\title{
A Real-Time Time-Dependent Density Functional Tight-Binding Implementation for Semiclassical Excited State Electron-Nuclear Dynamics and Pump-Probe Spectroscopy Simulations
}

\author{
Franco P. Bonafé, Bálint Aradi, Ben Hourahine, Carlos R. Medrano, Federico J. Hernández, \\ Thomas Frauenheim, and Cristián G. Sánchez*
}

Cite This: https://dx.doi.org/10.1021/acs.jctc.9b01217

Read Online

ABSTRACT: The increasing need to simulate the dynamics of photoexcited molecular systems and nanosystems in the subpicosecond regime demands new efficient tools able to describe the quantum nature of matter at a low computational cost. By combining the power of the approximate DFTB method with the semiclassical Ehrenfest method for nuclear-electron dynamics, we have achieved a real-time time-dependent DFTB (TDDFTB) implementation that fits such requirements. In addition to enabling the study of nuclear motion effects in photoinduced charge transfer processes, our code adds novel features to the realm of static and time-resolved computational spectroscopies. In particular, the optical properties of periodic materials such as graphene nanoribbons or

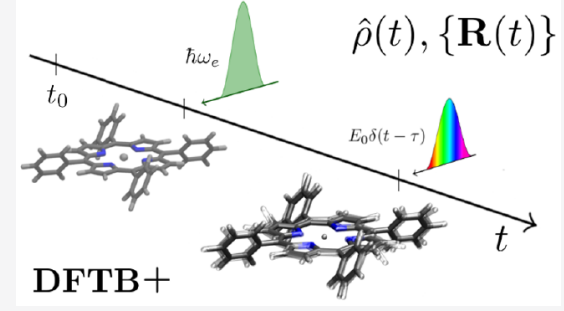
the use of corrections such as the "LDA+U" and "pseudo SIC" methods to improve the optical properties in some systems can now be handled at the TD-DFTB level. Moreover, the simulation of fully atomistic time-resolved transient absorption spectra and impulsive vibrational spectra can now be achieved within reasonable computing time, owing to the good performance of the implementation and a parallel simulation protocol. Its application to the study of UV/visible light-induced vibrational coherences in molecules is demonstrated and opens a new door into the mechanisms of nonequilibrium ultrafast phenomena in countless materials with relevant applications.

\section{INTRODUCTION}

Harnessing the excited state properties of molecules and nanostructures has become one of the main paths to successful solutions for technological challenges, ranging from artificial photosynthesis and solar cells to photocatalysis. It is also the core idea behind many fundamental lines of research in photochemistry, pump-probe spectroscopy, scanning tunneling microscopy, and so on. The large amount of research in these fields pushes the development of novel computational tools to efficiently perform simulations of these relevant processes. ${ }^{1,2}$ The most general solution would consist of solving the timedependent Schrödinger equation (TDSE) for coupled electronic and nuclei wave functions, thus considering both subsystems as quantum entities. However, this approach turns out to be too computationally demanding for many applications, and approximations have to be made.

Mixed quantum-classical approaches, which consider the nuclei as classical while keeping the electronic subsystem quantum, provide a way to achieve feasible simulations of excited state processes. The most commonly used trajectorybased methods are surface hopping and Ehrenfest dynamics. ${ }^{1-3}$ In the former, the potential energy surfaces (PES) of the system are calculated "on the fly", and in each trajectory, stochastic transitions between PES are allowed based on the flux of population between pairs of states, giving rise to the fewestswitches surface hopping (FSSH) method, one of its many variants. In FSSH, an ensemble of semiclassical trajectories must be run in order to compute the dynamics of interest. ${ }^{4}$ In Ehrenfest dynamics, on the other hand, the system is allowed to evolve in an average PES that results from a combination of all the occupied adiabatic states, in contrast to FSSH in which dynamics on a single PES is well described in uncoupled regions. $^{1}$

FSSH has been widely used to study transition probabilities along different paths to explain phenomena such as photochemical reactions, ${ }^{5}$ where the Ehrenfest method normally fails due to not allowing the system to hop between different PES. However, while Ehrenfest dynamics can be derived from first principles, by introducing the ansatz of a single product of the total wave function in the TDSE, ${ }^{1}$ the FSSH cannot be derived from first principles, although several quasi-derivations have been attempted.,

While several implementations of Ehrenfest dynamics have been published for model or atomistic systems, within different

Received: December 6, 2019

Published: June 8, 2020 
formalisms, there is a clear need for publicly available programs that propagate electron-nuclear trajectories for a wide range of atomistic systems using accurate yet efficient formalisms. Implementations of open source, free software offering realtime propagation of a time-dependent density functional theory (TD-DFT) scheme include the octopus code. ${ }^{8,9}$ Other implementations of Ehrenfest TD-DFT have also been reported. ${ }^{10,11}$ For some applications, however, it is useful to apply a more approximate formalism that, in turn, allows one to tackle very large systems or reach picosecond time scales at low computational cost. This enables applications such as the study of the optical properties of nanomaterials or biomolecules, ${ }^{12,13}$ the dynamics of optically excited vibrational modes in nanostructures, ${ }^{14}$ and other challenging systems. Real-time time-dependent density-functional tight binding (TD-DFTB) is a suitable formalism for these cases which has been widely used to calculate quantum-mechanically ground state static and dynamic properties of large atomistic systems. ${ }^{12,13,15-17}$

Here, we present the theoretical grounds and relevant computational details of a real-time Ehrenfest TD-DFTB implementation (available at https://github.com/dftbplus/ dftbplus) in the DFTB + code, $^{18}$ explained in Section 2. The possibility to calculate the optical properties of periodic systems that this implementation allows is discussed in Section 3.1 for graphene nanoribbons. The DFTB+ code not only provides an efficient and parallel way to calculate the ground state of molecular and periodic systems, i.e., the initial state for the dynamics, but also includes several corrections to the density functional tight-binding (DFTB) Hamiltonian. These include the approximate self-interaction corrections ("LDA+U" and "pseudo SIC" methods ${ }^{19}$ ) and third-order DFTB $^{20}$ (or "DFTB3"), which can be used to improve the modeling of time-dependent properties in some systems, as it is shown in Section 3.2. In Section 4, we discuss the application of this method to the calculation of the effect of the nuclei dynamics in the charge transfer profile in a donor-acceptor interface. The present implementation, within the limitations of Ehrenfest dynamics, is also able to simulate time-resolved transient absorption spectra and impulsive vibrational spectra, as far as we are aware, for the first time accounting for real-time propagation of the nuclei in an atomistic trajectory of several hundreds of femtoseconds. ${ }^{21}$ This development is presented in Section 5.1 and is applied to study the ultrafast dynamical features of on- and off-resonance excitation of zinc-tetraphenylporphyrin (ZnTPP) in Section 5.2. Finally, general conclusions and future prospects for this method are discussed in Section 6.

\section{THEORY AND IMPLEMENTATION DETAILS}

2.1. Theoretical Background. The Ehrenfest propagation scheme reported hereafter is based on the DFTB formalism. The DFTB method has been explained in detail in several references. $^{22-24}$ The following description summarizes the approximations of the method with respect to DFT. For simplicity, we consider the spin-unpolarized expressions, but the development has been done accounting for systems with collinear spin polarization. Starting from the energy expression as a functional of the density in DFT, $E[n]$, the second-order DFTB method is obtained by expanding the energy around a reference density $n_{0}$ up to second order in density fluctuations $\delta n^{23}$

$$
\begin{aligned}
E[n] & \approx \sum_{i} f_{i}\left\langle\psi_{i}\left|-\frac{1}{2} \nabla^{2}+V_{e x t}+V_{H}\left[n_{0}\right]+V_{x c}\left[n_{0}\right]\right| \psi_{i}\right\rangle \\
& +\frac{1}{2} \iint d \mathbf{r} d \mathbf{r}^{\prime}\left(\frac{\delta^{2} E_{x c}\left[n_{o}\right]}{\delta n \delta n^{\prime}}+\frac{1}{\left|\mathbf{r}-\mathbf{r}^{\prime}\right|}\right) \delta n \delta n^{\prime} \\
& -\frac{1}{2} \int V_{H}\left[n_{0}\right](\mathbf{r}) n_{0}(\mathbf{r})+E_{x c}\left[n_{0}\right]+E_{A A} \\
& -\int d \mathbf{r} V_{x c}\left[n_{0}\right](\mathbf{r}) n_{0}(\mathbf{r})
\end{aligned}
$$

where $\left|\psi_{i}\right\rangle$ are the molecular orbitals; $f_{i}$ is the occupation of the $\left|\psi_{i}\right\rangle$ state; $V_{e x t}, V_{H}$, and $V_{x c}$ are the external, Hartree, and exchange correlation potentials; $E_{x c}$ and $E_{A A}$ are the exchangecorrelation and internuclear repulsion energies, and $\mathbf{r}$ is the electronic position. A pseudoatomic orbital basis, $\left\{\left|\phi_{\mu}\right\rangle\right\}$, is considered, and using the two-center approximation for offdiagonal interactions, the matrix elements of the non selfconsistent Hamiltonian $H_{0}$ can be obtained and parametrized as a function of the interatomic distance. This leads to a simple expression for the first term of eq 1 (the non-SCC single particle energy)

$$
E_{\text {band }}=\sum_{i} f_{i}\left\langle\psi_{i}\left|H^{\circ}\right| \psi_{i}\right\rangle=\operatorname{Tr}\left\{\rho H^{\circ}\right\}
$$

where we have introduced the one-body reduced density matrix, $\rho$, and Tr represents the trace. The atomic contributions to the charge density are approximated by monopolar Mulliken charge fluctuations at the atoms. Assuming a spherical symmetry of the density fluctuation, the Coulomb integrals in the second term of eq 1 can be solved, and then, the Coulombic contribution is written as sums of products of atomic quantities

$$
\begin{aligned}
& E_{\text {coul }}=\frac{1}{2} \sum_{A B} \gamma_{A B} \Delta q_{A} \Delta q_{B} \\
& \gamma_{A B}= \begin{cases}U_{A}, & A=B \\
\frac{1}{R_{A B}}-\zeta\left(R_{A B}\right), & A \neq B\end{cases}
\end{aligned}
$$

where the parameters needed for the onsite terms (the Hubbard parameters $\left.U_{A}\right)$ and the short-range terms $\zeta\left(R_{A B}\right)$ are obtained from $a b$ initio calculations. The short-range term damps the Coulomb expression for short ranges and ensures the right limit at $R_{A B} \rightarrow 0$. The Mulliken populations are calculated as

$$
q_{A}=\sum_{\nu \in A} \sum_{\mu} \rho_{\nu \mu}\left\langle\phi_{\mu} \mid \phi_{\nu}\right\rangle=\operatorname{Tr}_{A}\{\rho S\}
$$

where $\operatorname{Tr}_{A}$ indicates the trace of over the orbitals centered on atom $A$, and the overlap matrix $S$ depends on the interatomic distance and is parametrized for each pair of orbitals. Beyondmonopole corrections have been previously developed and discussed for Slater-type-orbital-based DFTB ${ }^{25-27}$ and also for similar methods like Gaussian tight binding, ${ }^{28,29}$ which adds further contributions to the Coulomb terms of eq 3 and to the dipole matrix elements, which modifies directly the oscillator strengths. This is important to accurately describe the absorption spectra of molecules with low-lying $\sigma \rightarrow \pi^{*}$ and $n$ $\rightarrow \pi^{*}$ excitations. ${ }^{26}$ However, for most of the large molecular systems studied with the real-time Ehrenfest TDDFTB formalism, accurate results can be obtained in the monopole approximation at a very low computational cost. ${ }^{13-17,30,31}$ 
Hence, the monopole approximation has been used throughout this work.

The last four terms of eq 1 do not depend on the density fluctuation and hence can be collected into one single term, called the repulsion energy, $E_{r e p}$, which are decomposed into pairwise atomic contributions (neglecting single site terms, these being irrelevant for cohesive properties and relative energies), $V_{A B}^{r e p}$, that are also parametrized as a function of the interatomic distance. Hence, evaluation of the energy expression does not involve the computation of molecular integrals, which makes this approach extremely fast. The necessary parameters $H_{0}$ and $S$ are calculated in a minimal basis of valence orbitals for each pair of orbitals between each pair of atomic species at different interatomic distances. The repulsive terms, $V_{A B}^{r e p}$, are fitted minimizing DFTB and DFT energy and force differences for a set of systems that span a representative range between the pairs of atomic species $A$ and $B$. These are the only semiempirical parameters in the DFTB method. Several parameter sets including the ones used in the present work are available for download at http://www.dftb.org.

By minimizing the variation of the energy with respect to the expansion coefficients of the orthogonal molecular orbitals in the pseudoatomic basic, $\left\{C_{i \mu}\right\}$, given by $\left|\psi_{i}\right\rangle=\sum_{\nu} C_{i \mu}\left|\phi_{\mu}\right\rangle$, an expression for the DFTB Hamiltonian can be obtained

$$
H_{\mu \nu}=H_{\mu \nu}^{0}+\frac{1}{2} S_{\mu \nu} \sum_{C}\left(\gamma_{A C}+\gamma_{B C}\right) \Delta q_{C}, \quad \mu \in A, \nu \in B
$$

When an external potential is applied the Hamiltonian matrix changes to

$$
H_{\mu \nu} \rightarrow H_{\mu \nu}+\frac{1}{2} S_{\mu \nu}\left(V_{A}^{e x t}+V_{B}^{e x t}\right), \quad \mu \in A, \nu \in B
$$

The eigenvalue equation derived from the SCC Hamiltonian defined in eq 7 can be solved self-consistently to obtain the ground state (GS) of the system, by starting from a guessed charge distribution followed by consecutive cycles of diagonalization and Hamiltonian building until self-consistency. This method is known as self-consistent charge DFTB (SCC-DFTB).

Derivation of the equations of motion for wave function coefficients and nuclear positions according to the Ehrenfest method for tight-binding schemes have already been published ${ }^{32,33}$ and are well known from TD-DFT, where the timedependent Kohn-Sham equations can be obtained from Runge-Gross theorem. ${ }^{34}$ The equation of motion (EOM) of the coefficients $C_{i \mu}$ reads

$$
\dot{C}_{i \mu}=-\mathrm{i} \sum_{\kappa \nu} S_{\kappa \mu}^{-1}\left(H_{\kappa \nu}-\mathrm{i}\left\langle\phi_{\kappa} \mid \dot{\phi}_{\nu}\right\rangle\right) C_{i \nu}
$$

All the matrix elements on the right-hand side have already been introduced throughout this text, except for the nonadiabatic coupling (NAC) matrix elements

$$
D_{\kappa \nu}=\left\langle\phi_{\kappa} \mid \dot{\phi}_{\nu}\right\rangle
$$

As the pseudoatomic basis set is centered on the atomic nuclei, these matrix elements are nonzero only when the nuclei move. The EOM of the density matrix can be obtained, as shown in the Appendix A, from eq 8

$$
\dot{\rho}=-\mathrm{i}\left(S^{-1} H \rho-\rho H S^{-1}\right)-\left(S^{-1} \mathrm{D} \rho+\rho D^{\dagger} S^{-1}\right)
$$

The first term of the right-hand side of this equation is the electronic EOM known as the Liouville-von Neumann equation, $^{35}$ a generalized commutator in a nonorthogonal basis, and it has been implemented and extensively used for the calculation of optical properties of materials and time-dependent photoinduced phenomena keeping the nuclei "clamped". 12,13,15-17,31,36 The second term gives rise to the nonadiabatic interactions, allowing energy exchange between electrons and nuclei at the Ehrenfest level.

As the basis orbitals' time evolution is tied to the nuclei on which they are centered, the NAC elements can be computed from the chain rule. By making explicit the nuclear index where each orbital is centered, the $D$ matrix elements are then

$$
D_{\mu \nu}=\left\langle\phi_{\mu} \mid \dot{\phi}_{\nu}\right\rangle=\left\langle\phi_{\mu} \mid \nabla_{B} \phi_{\nu}\right\rangle \cdot \dot{\mathbf{R}}_{B}, \quad \mu \in A, \nu \in B
$$

where $\nabla_{B}$ is the gradient applied with respect to the coordinates of atom $B$, and $\dot{R}$ is the velocity. To calculate $\left\langle\phi_{\mu} \mid \nabla_{B} \phi_{\nu}\right\rangle$, we use the fact that $S_{\mu \nu}$ is only a function of $\mathbf{R}_{A}-\mathbf{R}_{B}$, and hence, for orbitals sitting at different atoms $A \neq B$

$$
\nabla_{B} S_{\mu \nu}=\left\langle\nabla_{B} \phi_{\mu} \mid \phi_{\nu}\right\rangle+\left\langle\phi_{\mu} \mid \nabla_{B} \phi_{\nu}\right\rangle=\left\langle\phi_{\mu} \mid \nabla_{B} \phi_{\nu}\right\rangle
$$

since $\nabla_{B} \phi_{\mu}=0$. This result is equivalent to the one derived in ref 32. Formally, for orbitals sitting on the same atom (onsite blocks of $D$ ), the limit of the gradient in eq 12 as the two atoms approach each other must be taken

$$
\left\langle\phi_{\mu_{A}} \mid \nabla_{A} \phi_{\nu_{A}}\right\rangle=\lim _{\left|\mathbf{R}_{A^{\prime}}-\mathbf{R}_{A}\right| \rightarrow 0} \nabla_{A} S_{\mu_{A^{\prime}} \nu_{A}}
$$

Considering that the $D$ matrix elements can be related to the linear momentum matrix elements, ${ }^{32}$ the onsite elements can be regarded as the small internuclear distance limit of an atomic scattering problem. This way it can be argued that the onsite blocks can been set to zero. ${ }^{37}$ This has been used and validated in previous Ehrenfest implementations ${ }^{33}$ and in this implementation by comparison with reported light-induced impulsive vibrations. ${ }^{14,21}$ The following general expression for the NAC matrix elements can then be written

$$
D_{\mu \nu}=\dot{\mathbf{R}}_{B} \cdot \nabla_{B} S_{\mu \nu}\left(1-\delta_{A B}\right)
$$

From eq 14, two conclusions can be drawn:

(a) For fixed nuclei, $D=0$, and the electronic EOM, the first term in eq 10 , is recovered.

(b) It can be verified that $D^{\dagger} \neq-D$. If they were equal, this would allow the effective Hamiltonian $\tilde{H}$ to be hermitian (see Appendix A), making the evolution unitary, with no change in the eigenvalues of the density matrix. This only happens when all velocities are equal, which is the case for center of mass motion only without rotation. Hence, unitary evolution is recovered when there is no relative motion of the atoms.

The EOM for the nuclei has also been derived in previous works, starting from the Lagrangian of the system. ${ }^{32,33}$ The Euler-Lagrange equations for the coefficients lead to the EOM for the coefficients, eq 8, while differentiating the Lagrangian with respect to the nuclear coordinates leads to the expression for the Ehrenfest force. Rewritten here in terms of the density matrix, this is 


$$
\begin{aligned}
& \mathbf{F}_{A} \\
& \quad=-\operatorname{Tr}\left\{\rho\left(\nabla_{A} H^{\circ}+\nabla_{A} S \sum_{B} \gamma_{A B} \Delta q_{B}+\left(\nabla_{A} S\right) S^{-1} H+H S^{-1}\left(\nabla_{A} S\right)\right)\right\} \\
& -i \operatorname{Tr}\left\{\rho\left(\nabla_{A} S\right) S^{-1} D+\text { h.c. }\right\}+\mathrm{i} \sum_{\mu \nu}\left\{\rho_{\nu \mu}\left\langle\nabla_{A} \phi_{\mu} \mid \nabla_{B} \phi_{\nu}\right\rangle \cdot \dot{\mathbf{R}}_{B}+\text { h.c. }\right\} \\
& -\Delta q_{A} \sum_{B} \nabla_{A} \gamma_{A B} \Delta q_{B}-\nabla_{A} E_{r e p}-\Delta q_{A} \mathbf{E}(t), \quad \mu \in A, \nu \in B
\end{aligned}
$$

where $\mathbf{E}(t)$ is the time-dependent external field, h.c. is the hermitian conjugate, and the second and third terms depend on the nuclear velocities, which cancel each other out for a complete basis. ${ }^{33}$ These terms are necessary for momentum conservation but not for energy conservation, since the latter emerges from the lack of explicit time dependence of the Lagrangian, nor do they produce net work. ${ }^{32}$ Total charge conservation, however, is given by the velocity-dependent terms in the electronic EOM, which are indeed included (namely, the NAC terms). In the present implementation, the velocitydependent terms of the nuclear forces are ignored, since for all the cases of interest considered here these terms are negligible due to the small nuclear velocities involved. Moreover, the fact that the forces depend only on the electronic state and the nuclear positions enables more efficient methods for propagating the nuclei. Otherwise, other methods typically used for dynamics with dissipative forces must be used, where the forces and velocities must be solved self-consistently at each time step.

To drive the system, a time-dependent external field can be added to the DFTB Hamiltonian according to eq 7, where the external potential can be calculated under the electric dipole approximation

$$
V_{A}^{\text {ext }}(t)=-\boldsymbol{\mu}_{A}(t) \cdot \mathbf{E}(t)=\Delta q_{A}(t) \mathbf{R}_{A}(t) \cdot \mathbf{E}(t)
$$

The limitations of the Ehrenfest method, such as its inability to reproduce Joule heating on the nanoscale ${ }^{38}$ or the photoisomerization of retinal models, ${ }^{33}$ are well known. The lack of nuclear fluctuations suppresses heat dissipation to the nuclei from excited electrons. ${ }^{38}$ To observe inelastic dissipation, other methods such as lowest-order electron-phonon scattering theory are needed, where the electron-phonon interaction is treated by first-order perturbation theory. ${ }^{39}$ This theory however fails in the strong electron-phonon coupling regime, and other methods such as the self-consistent Born approximation $^{40}$ or the coupled electron ion dynamics (CEID) method $^{41}$ are necessary.

On the other hand, the method is fully quantum coherent, since the nondiagonal elements of the DM (coherences) are included in the dynamics. Besides, it has been proven that the nuclear motion, through its effect in the Hamiltonian, can induce electronic transitions, mainly in metals, and can lead to an electronic state that can be characterized by a temperature. ${ }^{42}$ However, although the electrons achieve a Fermi-Dirac distribution, its temperature is different from that of the nuclei and hence cannot be regarded as an equilibration process.

2.2. Ground State Absorption Spectra. The simulation protocol to compute the GS absorption has been derived two decades ago and has been used in several research works by different groups. This short summary highlights the main concepts and is useful in Section 5.1. First, the ground state DM $\rho_{0}$ is kicked by a Dirac-delta electric field pulse $\mathbf{E}_{\text {kick }}(t)=\mathcal{E}_{0} \delta(t) \hat{e}$ polarized in direction $\hat{\mathbf{e}} \in\{\hat{i}, \hat{j}, \hat{k}\}$, exciting all dipole allowed transitions. ${ }^{43}$ For a nonorthogonal basis set, this DM immediately after a Dirac-delta perturbation is calculated according to eq $16^{44}$

$$
\rho\left(t=0^{+}\right)=\frac{1}{2}\left(e^{i / \hbar \hat{V}} \rho_{0} S e^{-i / \hbar \hat{V}} S^{-1}+S^{-1} e^{i / \hbar \hat{V}} S \rho_{0} e^{-i / \hbar \hat{V}}\right)
$$

where $\hat{V}=-\hat{\boldsymbol{\mu}} \cdot \mathbf{E}_{\text {kick }}$. The system then evolves freely, and the time-dependent dipole moment of the system contains the information about the excited frequencies and oscillator strengths. By Fourier transforming the dipole moment signal along each Cartesian direction after exciting in any other Cartesian direction, the polarizability tensor (Fourier transform of the response function) for all angular frequencies $\omega$ can be found $^{45}$

$$
\boldsymbol{\alpha}(\omega)=\frac{\boldsymbol{\mu}(\omega)-\boldsymbol{\mu}_{0}}{\mathcal{E}_{0}}
$$

where $\mathcal{E}_{0}$ is the Dirac-delta (scalar) field strength. The absorption cross section for randomly oriented molecules can be calculated as ${ }^{46}$

$$
\sigma(\omega)=\frac{4 \pi \omega}{c} \operatorname{Im}(\bar{\alpha}(\omega))
$$

where $\bar{\alpha}(\omega)=1 / 3 \operatorname{Tr}[\boldsymbol{\alpha}(\omega))]$. This method has been used by some of us to calculate absorption spectra of molecular systems, ${ }^{15,17,31}$ semiconductors, ${ }^{16,36,47,48}$ and metallic nanoparticles, ${ }^{12,49}$ as well as carbon-based nanostructures. ${ }^{13}$

2.3. Implementation. The implementation takes advantage of the GS and structural relaxation currently implemented in DFTB + . The time evolution is achieved by time discretization and numerical propagation of the DM and nuclear coordinates in the time grid. The integration of the density matrix is carried out using the Leapfrog technique, a second-order method, given by the following update equation

$$
\rho_{i+1}=\rho_{i-1}+2 \Delta t \dot{\rho}_{i}
$$

where $\dot{\rho}_{i}$ is calculated using eq 10 . The integration step that ensures stable dynamics for most systems is between 1 and 5 . As it is a two-step method, it must be initialized using a single-step method to compute the initial states for propagation; here, the Euler method is used. The bottleneck of this method is given by the calculation of $\dot{\rho}$ which can be done in three matrix-matrix multiplications, computed here using optimized BLAS subroutines and with a maximum scaling with the size of the system (number of basis orbitals $N$ ) of $O\left(N^{3}\right)$. This method has the advantage over other choices such as the Crank-Nicholson or Magnus expansion that it only needs the Hamiltonian at the present time step to compute the DM at the following time step, so no extrapolation or predictor-corrector scheme is necessary. Moreover, it exactly conserves the number of electrons and is time reversible.

The NAC matrix elements are calculated using eq 14 where the gradients of $S$ are approximated by finite differences. The derivative with respect to atom $B$ in the direction $\alpha$ is given by

$$
\frac{\partial}{\partial R_{B_{\alpha}}} S=\frac{1}{2 \Delta R}\left(S\left(R_{A B_{\alpha}}+\Delta R\right)-S\left(R_{A B_{\alpha}}-\Delta R\right)\right)
$$

Here, $\mathbf{R}_{A B}=\mathbf{R}_{B}-\mathbf{R}_{A}$ and $\Delta R=\sqrt[4]{\epsilon}$, where $\epsilon$ is the double precision machine epsilon. $\Delta R$ is chosen since the rounding error for a step of size $h$ is of the order of $\epsilon / h^{2}$, and the central difference derivative formula has a truncation error of $O\left(h^{2}\right)$. 


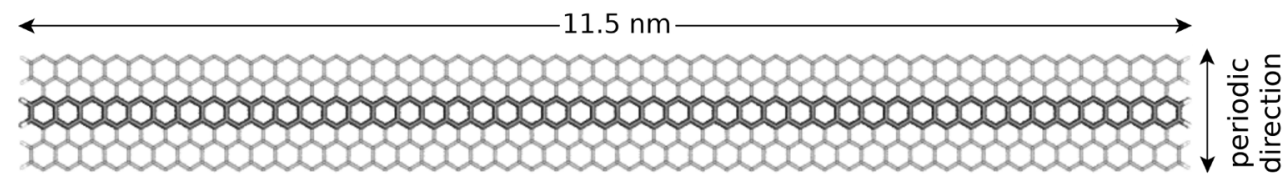

(a)

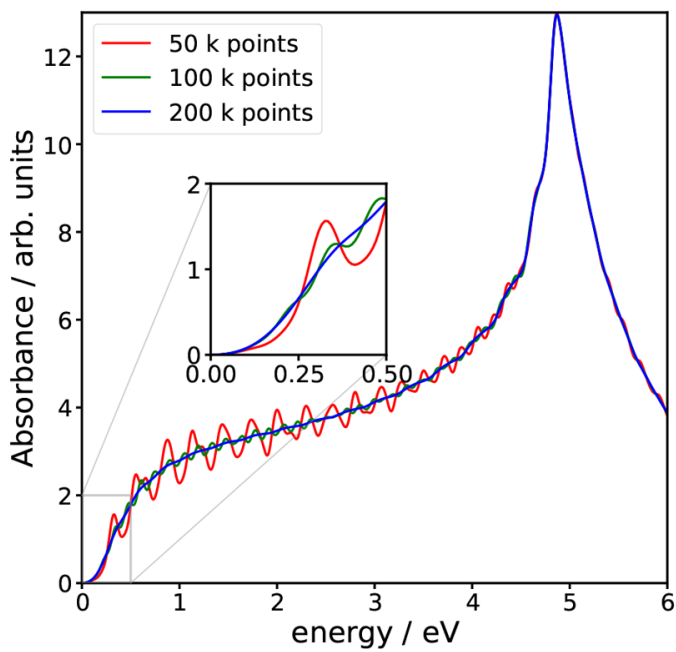

(b)

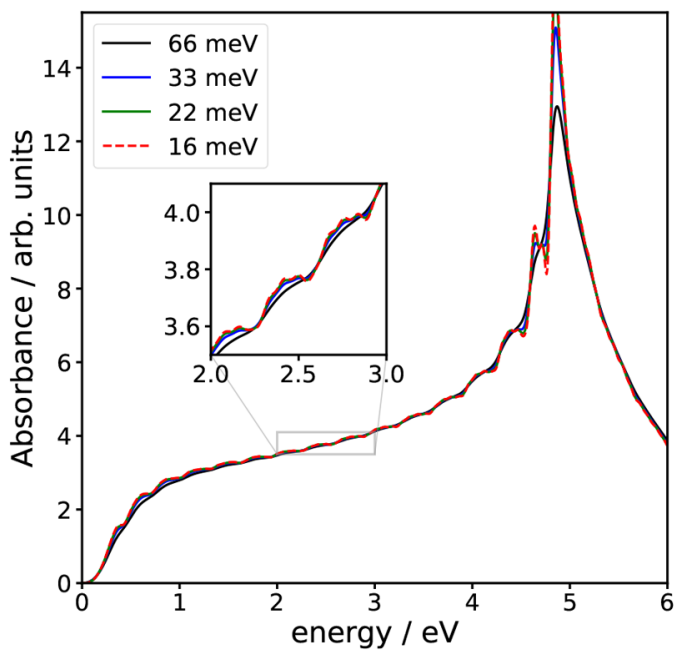

(c)

Figure 1. (a) Unit-cell representation for the 95-aGNR studied. (b) Electronic spectra in the direction normal to the ribbon axis for the semiconducting 95 -aGNR of the $3 p+2$ family using different numbers of $\mathbf{k}$ points to sample the periodic direction of the first Brillouin zone. A broadening of $66 \mathrm{meV}$, corresponding to an exponential damping decay time of $10 \mathrm{fs}$, was used in all cases. (c) Same as (b) for different broadenings. $200 \mathrm{k}$-point samples within the first Brillouin zone in the periodic direction were used in all cases.

The forces (eq 15) are computed using the sparse matrix subroutines included in DFTB,$+{ }^{50}$ using numerical approximations for $\nabla_{A} H$ and $\nabla_{A} S$ and the analytical expressions for $\nabla_{A} \gamma_{A B}$ and $\nabla_{A} E_{\text {rep. }}$. The propagation of the nuclear coordinates uses the well-known velocity Verlet method. ${ }^{51}$

\section{NOVEL SIMULATIONS OF OPTICAL PROPERTIES}

3.1. Optical Properties of Graphene Nanoribbons. After the experimental synthesis of graphene by Novoselov in $2004,{ }^{52}$ it has been considered a promising material for future electronics. Due to its extremely high charge-carrier mobilities, ${ }^{52,53}$ graphene-based transistors have been developed rapidly and are considered as a choice for postsilicon electronics. ${ }^{54}$ However, the lack of a bandgap in graphene hinders its application in field-effect transistors devices. ${ }^{54}$ In this sense, flat one-dimensional $\mathrm{sp}^{2}$ carbon-based materials, known as graphene nanoribbons (GNRs), are proposed as the building blocks for the next generation in this field. Unlike infinite graphene, GNRs show nonzero bandgaps that strongly depend on the ribbon width. ${ }^{55-57}$ Particularly, armchair GNRs (aGNRs) are the most promising candidates in this area because of the high tunability of their electronic properties. ${ }^{57}$ In this context, the study of the optoelectronic properties of these kinds of structures is necessary in order to drive the development of new carbon-based nanoelectronic technologies.

The absorption spectrum of a 95 atom wide arm-chair graphene nanorribon (95-aGNR, see Figure 1a) was calculated as described in Section 2.2 as an example of the possibility to calculate spectra of lower-dimensional structures. The mio-1-1 Slater-Koster parameter set was used. ${ }^{58}$ The absorption spectrum for light polarized in the plane of the ribbon and normal to the periodic direction is shown in Figure 1 for different $\mathbf{k}$-point samplings and signal damping times. The calculated absorption shows an optical gap of about $10 \mathrm{meV}$, obtained by linear extrapolation of the absorption close to the gap. This small gap is expected for an armchair nanorribon of the $3 p+2$ family. ${ }^{59}$ The absorption raises rapidly with energy between 0 to $1 \mathrm{eV}$ and then slowly to about $4 \mathrm{eV}$. A sharp peak is present in the absorbance at $5 \mathrm{eV}$ which is about five times higher than the absorption at $2 \mathrm{eV}$. These features are in good agreement with existing results in the literature ${ }^{60}$ obtained using a very similar method to the one present in this work but based on time-dependent DFT. The spectra shown in Figure 1 are also in good agreement to the calculations presented by Yang et al. ${ }^{61}$ for graphene not including excitonic effects. When compared to the results in ref 61 that include excitonic effects, the peak at $5 \mathrm{eV}$ in Figure 1 is at higher energies but lower than those shown by Lian et al. in ref 60 . The fact that results using TD-DFTB improve upon those of TD-DFT has been observed by some of us before ${ }^{31}$ and clearly corresponds to a compensation of errors arising from the model.

Regarding the large number of k-point samples that are required to converge absorption spectra, as can be seen from Figure $1 b$, a large density of points is required. This is to be expected as a similar density of sampling would be needed to obtain a converged density of states. Once the spectrum is converged with respect to sampling of the Brillouin zone, as depicted in Figure 1c, the spectrum is independent of the broadening or damping used before the Fourier transform of the time dependent dipole moment. 
3.2. DFTB+U spectra for $\mathrm{Ni}(\mathrm{CO})_{4}$. For systems where there is a strong interaction between localized electrons, such as transition metal or lanthanide compounds and solids, a simple mean-field correction for electron correlation is provided by the LDA+U family of methods. ${ }^{64}$ The rotationally invariant ${ }^{65}$ form of $\mathrm{LDA}+\mathrm{U}$ can be written in terms of several choices of local projections of the density matrix. ${ }^{66}$ Likewise, the doublecounting between the Hubbard model and the density functional mean-field functional takes several limiting cases. ${ }^{67}$ In the DFTB+ code, the dual population projected form of the fully localized limit (FLL) functional has been available for ground state calculations for over a decade. ${ }^{68}$ The additions to the DFTB energy and Hamiltonian take the form

$$
\begin{aligned}
& \Delta E^{F L L}=-\sum_{A} \sum_{l} \sum_{\mu \nu} \frac{U_{l}^{\mathrm{eff}}}{2}\left(n_{\mu \nu}^{2}-n_{\mu \nu}\right) \\
& \Delta V_{\mu \nu}^{F L L}=-U_{l}^{\mathrm{eff}} S_{\mu \nu}\left(n_{\mu \nu}-\frac{1}{2} \delta_{\mu \nu}\right) \\
& n_{\mu \nu}=\frac{1}{2} \sum_{\mu \nu} \sum_{\kappa}\left(\rho_{\mu \kappa} S_{\kappa \nu}+S_{\mu \kappa} \rho_{\kappa \nu}\right)
\end{aligned}
$$

where $\{\mu, \nu\} \in l \in A$, i.e., the orbitals within the angular shell $l$ on atom $A$, but $\kappa$ is summed over all orbitals for which the overlap to that atomic block is nonzero.

The choice of the effective Hubbard- $U$ parameter, $U^{\text {eff }}$, is a contentious issue with a number of recipes in the literature, ranging from an empirical choice to reproduce experiments to recipes for screened self-consistent evaluation. Figure 2 shows

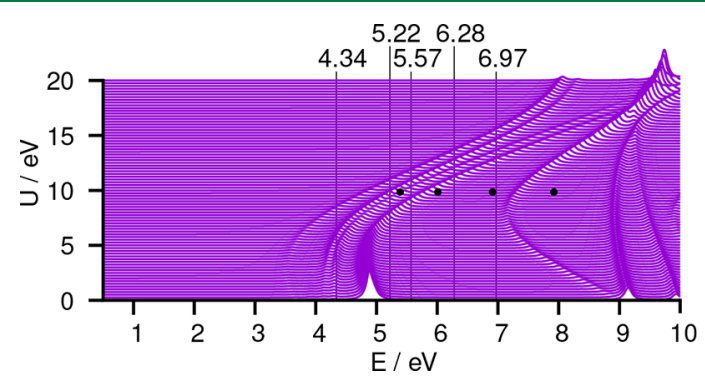

Figure 2. Electronic spectra of $\mathrm{Ni}(\mathrm{CO})_{4}$ for ground state relaxed DFTB $+\mathrm{U}$ geometries. The marked circles correspond to TD-GGA+U results, ${ }^{62}$ with the CASPT2 results of ref 63 marked along the top of the figure.

the excitation energies of gas phase $\mathrm{Ni}(\mathrm{CO})_{4}$ from one selfconsistent GGA+U approach, ${ }^{62}$ marked as circles for the value of $U^{\text {eff }}$ and the resulting optically active transition energies. This figure also shows the results of correlated wave function-based calculations. ${ }^{63}$ These are compared against DFTB+U (the purple traces) for a range of $U^{\text {eff }}$ values from uncorrelated $\left(U^{\text {eff }}=\right.$ $0)$ to approximately twice the unscreened atomic values for $\mathrm{Ni}^{3 \mathrm{~d}}$ at the GGA level $(20 \mathrm{eV})$. The trans $3 \mathrm{~d}^{69}$ and mio ${ }^{58}$ SlaterKoster parameter sets were used, where the shell-resolved form of the DFTB Hamiltonian was applied (as a result the mio C and $\mathrm{O}$ parameters were edited to set atomic Hubbard-U values to their highest atomic occupied values, correcting the known error in these parameters when used for shell resolved calculations). The DFTB+U absorption spectra were obtained by optimizing the GS geometry at each value of $U$ and using this as the source of the initial density matrix. The absorption spectra were then calculated for a fixed geometry by applying the approach of
Section 2.2 using the DFTB+U Hamiltonian to propagate the density matrix.

The closest match to the CASPT2 results is obtained for an effective $U_{3 d}$ value of $\sim 8 \mathrm{eV}$, where the $\mathrm{DFTB}+\mathrm{U}$ features most closely approach the excitation energies of this method. This is slightly lower than the $9.85 \mathrm{eV}$ effective $U$ determined selfconsistently for GGA. This is primarily due to the lower-most and particularly the upper-most absorption features in the $\mathrm{DFTB}+\mathrm{U}$ spectra, which most closely approach the CASPT2 features at a lower value of $U$. The three central features in the group instead best match these results for a value of $U$ slightly above $10 \mathrm{eV}$, leading to the effective value for best spectroscopic properties on average. Unfortunately, it is not at present possible to verify the symmetry assignments of the modes with real time propagation methods (since the density matrix transforms with the full symmetry of the Hamiltonian, apart from the spin and the effects of spin contamination ${ }^{70}$ ). But the recent work of Oppenheim et al. ${ }^{71}$ demonstrates that the symmetries of the excitations in a related nickel compound are correctly reproduced for a range of density-matrix functionals.

Pseudo-SIC ${ }^{72}$ instead approximately removes the local part of the self-interaction error but only affects the occupied states of the system, unlike FLL which is similar to a scissor operation on the affected shell. Again, this can be expressed in the local block populations

$$
\begin{aligned}
& \Delta E^{F L L}=-\sum_{A} \sum_{l \in A} \frac{\tilde{U}_{l}^{\mathrm{eff}}}{2} n_{l}^{2} \\
& \Delta V_{l}^{F L L}=-\tilde{U}^{\mathrm{eff}} S_{l} n_{l}
\end{aligned}
$$

where $\tilde{U}$ is approximately $1 / 4$ of the atomic $U$ values once relaxation is taken into account. ${ }^{19,73} \mathrm{We}$ find a best match to the CASTPT2 results for $\tilde{U}_{3 d} \approx 4.6 \mathrm{eV}$ with this approximation, with qualitatively similar features to the FLL results.

\section{CHARGE TRANSFER IN A NANODIAMOND DONOR-ACCEPTOR COMPLEX}

One exciting application that this implementation enables is the study of the influence of the nuclear motion on light-induced charge transfer processes in donor-acceptor complexes. These kinds of systems are the building blocks for optoelectronic materials for light to electricity conversion devices as dyesensitized solar cells or organic solar cells. Recent experimental and theoretical studies show that coherent vibronic coupling between electrons and nuclei is of key importance for the description of the first steps of charge separation in noncovalent $^{74}$ and covalently ${ }^{75}$ linked organic systems and also in vertically stacked transition-metal dichalcogenide layers. ${ }^{76,77}$ These works reveal the increasing need to take into account the nuclear motion to describe the charge transfer processes in such materials.

In a previous work, we have studied the photodynamic process in a supramolecular arrangement composed of a hydrogenated nanodiamond $\left(\mathrm{C}_{190} \mathrm{H}_{110}\right)$ interacting with a 3,4,9,10-perylenetetracarboxylic acid diimide (PDI) molecule as acceptor. After pulse irradiation in tune with the photoexcitation of the acceptor, the system shows an ultrafast charge transfer process reaching a stable steady state in a few tens of femtoseconds. We proposed a purely electronic reordering of the system after charge separation as the reason for the irreversibility of the process. To date, we had not considered the 


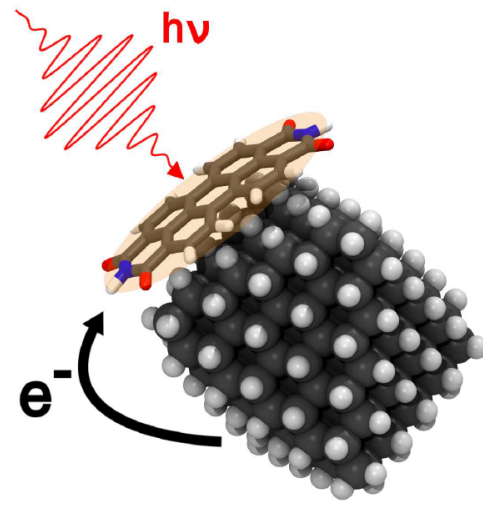

(a)

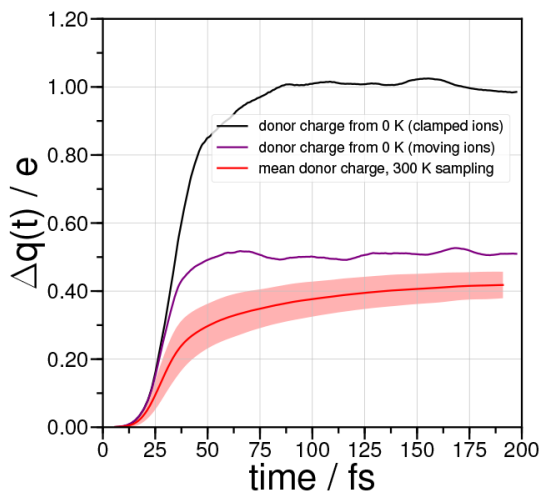

(b)

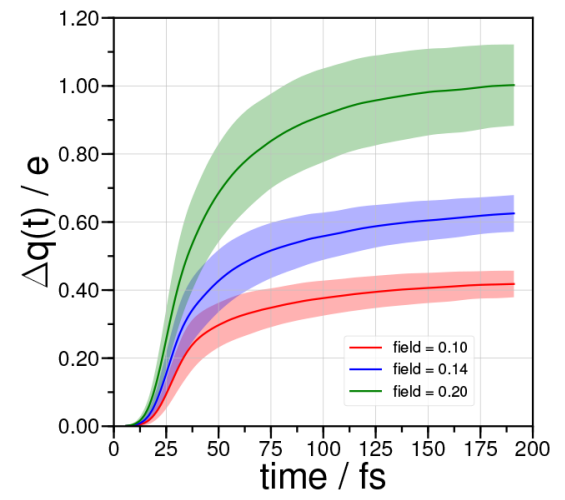

(c)

Figure 3. (a) $\mathrm{C}_{190} \mathrm{H}_{110}+\mathrm{PDI}$ donor-acceptor complex representation. The system is composed of a perylene tetracarboxylic diimide (acceptor) interacting with a hydrogen-terminated ND (donor) by London dispersion forces. The figure describes qualitatively the photodynamic process of charge transfer. (b) Donor charge as a function of time after $50 \mathrm{fs}$ pulse irradiation tuned with the acceptor lower-energy excitation. The red and blue curves indicate fixed (frozen) ions and moving ions, respectively.

nuclear motion for the description of this "trap-door"-like mechanism.

On the basis of our experience of how systems react to sudden changes in electronic structure, we expect coherent breathing oscillations to be launched impulsively in the nanodiamond. ${ }^{14}$ Here, we show some preliminary simulations of the same system presented in ref 78 (see Figure 3a) including the nuclear motion using the Ehrenfest TD-DFTB method described in the Section 2.

Figure $3 \mathrm{~b}$ shows the evolution of the Mulliken charge distribution in the ND (donor) upon the photoexcitation of the complex with a pulsed electric field perturbation in tune with the acceptor excitation energy. The black and purple curves represent the dynamics considering clamped and moving ions, respectively. The nuclear motion decreases the total steady state charge transfer by half. To take into account the effect of the temperature, we have also performed Ehrenfest dynamics starting from configurations sampled at $300 \mathrm{~K}$ from an NVT ensemble by means of Born-Oppenheimer molecular dynamics performed using the DFTB+ package ${ }^{18}$ (see Supporting Information for details). Figure $3 \mathrm{~b}$ also shows the mean charge of the donor obtained from averaging the simulations for 15 decorrelated configurations (red curve), together with its standard deviation. Higher temperatures also decrease the net charge transfer, but with a less pronounced effect.

To find whether the nuclear motion limits the total charge transferred, we have also studied its dependence on the external field strength. As shown in Figure 3c, charge transfer can be enhanced by using a stronger external field, reaching a total charge transfer of $1 \mathrm{e}$ as in the case of fixed nuclei by increasing the field strength from $0.1 \mathrm{~V} / \AA ̊$ to $0.2 \mathrm{~V} / \AA$.

In summary, under the same illumination conditions, a strong decrease in the charge transfer is observed when nuclear motion is allowed, supporting our previous hypothesis. ${ }^{78}$ This can be interpreted as follows: nuclear motion increases the detuning, which allows the system to reach the steady state regime sooner. This, in turn, reduces the total charge transferred, since a new relaxation channel (i.e., the nuclear degrees of freedom) is added. However, nuclear motion and temperature do not affect the trap-door-like mechanism, as the system still reaches a steady state after the pulse perturbation. These preliminary results show that nuclear motion is not negligible even at very short time scales, having an effect in ultrafast charge transfer mechanisms.

\section{TRANSIENT ABSORPTION SPECTRA SIMULATIONS}

5.1. Theory and Computational Method. One of the most novel applications of the present implementation is the simulation of time-resolved pump-probe spectroscopy experiments in the UV/visible range. Within these techniques, in particular, broadband time-resolved transient absorption (TA) spectroscopy is one of the most widely used and can be straightforwardly obtained using the Ehrenfest real time TDDFTB method, up to its limitations, as is shown below.

To measure broadband TA spectra, a system is first excited using a pump pulse tuned to a specific wavelength. After a delay time $\tau$ has elapsed, a weaker probe pulse is applied, and the absorption at all wavelengths within the probe bandwidth, $A(\omega$, $\tau)$, is obtained. This procedure can be repeated to measure the absorption at different delay times, building a time-resolved absorption. The quantity of interest is the normalized differential transmittance, $\Delta T / T$, or the transient absorbance, $\Delta A(\omega$, $\tau)=A(\omega, \tau)-A_{0}(\omega)$, which is connected via $\Delta A=-\Delta T / T$ if $\Delta T \ll T_{0}$, with $T_{0}$ being the transmittance of the unperturbed system.

Several theoretical models have been developed since the 1990s to extract quantitative information about the potential energy surfaces that drive the dynamics of the system. ${ }^{79}$ In nonequilibrium spectroscopy, the nonlinear terms of the response function (or susceptibility) of the system are relevant to compute the total polarization of the system. In isotropic media, the even-order response functions vanish due to the inversion symmetry, thus being the third-order response function of the first nonlinear term in most materials. This term can be represented as a triple commutator of the dipole moment in the interaction picture with the equilibrium DM, which gives eight terms upon expansion. ${ }^{80}$ These eight terms represent different time ordering of the interactions with the external field. For the case of TA spectra, only six of these terms survive. Following this approach and fitting parameters for model systems, usually with few (more than three) electronic levels and one or two nuclear coordinates, several simulations of TA spectra have been done. ${ }^{79,81-90}$ This framework, however, is not practical when a mean field Hamiltonian (like in DFT or 
DFTB) is used, since the complexity of the third-order response function grows very fast with the size of the system $\left(O\left(N^{16}\right)\right)$.

A different approach suitable for $a b$ initio or semiempirical atomistic simulations is based on the real-time propagation of the full molecular or nanostructured system. The work by De Giovannini et al. introduced the idea of using the pumpdependent effective response function $\chi_{\text {eff }}$ of the system within real-time TD-DFT for the calculation of TA spectra with pulses in the attosecond regime and without considering nuclear motion due to the short time scales. ${ }^{91}$ Following a similar idea, Boleininger was able to compute the transient core absorption spectra of polythiophene fragments over various delay times of up to $\sim 20$ fs using Ehrenfest time-dependent Gaussian tightbinding and XUV probe pulses. ${ }^{92}$

To derive an expression that provides more insight on the structure of the $\chi_{\text {eff }}$, we start by considering a simulation protocol similar to the one used to compute the ground state spectra described in Section 2.2. We aim to derive a method that allows us to use the time-dependent dipole moment, $\boldsymbol{\mu}(t)$, after probing the system with a kick $\mathbf{E}_{k i c k}$, subtracting all other contributions that are not frequencies excited by the probe, hereafter referred to as reference dipole moment $\boldsymbol{\mu}_{\text {ref }}(t)$

$$
\boldsymbol{\mu}(t)-\boldsymbol{\mu}_{r e f}(t)=\int_{-\infty}^{\infty} \chi_{e f f}(t, \tau) \mathbf{E}_{k i c k}(\tau) d \tau
$$

Such a system has been driven out of the equilibrium by a pump pulse, represented as

$$
\mathbf{E}_{b}(t)=\mathcal{E}_{0}^{b} \sin (\omega t+\phi) f(t) \hat{\mathbf{e}}_{b}
$$

where $\mathcal{E}_{0}^{b}$ is the peak intensity; $\omega, \phi$, and $f(t)$ are the carrier frequency, phase, and envelope function; and $\hat{\mathbf{e}}_{b}$ the polarization direction. The pump pulse total duration is $t_{p}$, and for simplicity, we consider that it is nonzero only, $0 \leq t \leq t_{p}$.

The expansion of the dipole moment in terms of the pump field is given by

$$
\begin{aligned}
& \boldsymbol{\mu}_{b}(t)=\boldsymbol{\mu}_{0}^{b}+\int \chi^{(1)}(t, \tau) \mathbf{E}_{b}(\tau) d \tau \\
& +\frac{1}{2 !} \iint \chi^{(2)}\left(t, \tau, \tau^{\prime}\right) \mathbf{E}_{b}(\tau) \mathbf{E}_{b}\left(\tau^{\prime}\right) d \tau d \tau^{\prime} \\
& \quad+\frac{1}{3 !} \iiint \chi^{(3)}\left(t, \tau, \tau^{\prime}, \tau^{\prime \prime}\right) \mathbf{E}_{b}(\tau) \mathbf{E}_{b}\left(\tau^{\prime}\right) \mathbf{E}_{b}\left(\tau^{\prime \prime}\right) d \tau d \tau^{\prime} d \tau^{\prime \prime} \\
& \quad+\ldots
\end{aligned}
$$

where $\boldsymbol{\mu}_{0}^{b}$ is the static dipole moment, and $\chi^{(n)}$ is the $n$th order response function. Since the pump pulse is strong, this expansion cannot be truncated to first order, so all orders must be considered. Now, consider that at $t=t_{s}$, the probe pulse is applied, with the aforementioned Dirac-delta shape

$$
\mathbf{E}_{s}(t)=\mathcal{E}_{0}^{s} \delta\left(t-t_{s}\right) \hat{e}_{s}
$$

where $\mathcal{E}_{0}^{s}$ and $\hat{\mathbf{e}}_{s}$ have the equivalent meaning as in the pump pulse. Hence, the total applied field is $\mathbf{E}(t)=\mathbf{E}_{b}(t)+\mathbf{E}_{s}(t)$. The dipole response for the total field is then

$$
\begin{aligned}
& \boldsymbol{\mu}(t)=\boldsymbol{\mu}_{0}+\int \chi^{(1)}(t, \tau)\left(\mathbf{E}_{b}(\tau)+\mathbf{E}_{s}(\tau)\right) d \tau \\
& +\iint \chi^{(2)}\left(t, \tau, \tau^{\prime}\right)\left(\mathbf{E}_{b}(\tau)+\mathbf{E}_{s}(\tau)\right)\left(\mathbf{E}_{b}\left(\tau^{\prime}\right)+\mathbf{E}_{s}\left(\tau^{\prime}\right)\right) d \tau d \tau^{\prime} \\
& +\iiint \chi^{(3)}\left(t, \tau, \tau^{\prime}, \tau^{\prime \prime}\right)\left(\mathbf{E}_{b}(\tau)+\mathbf{E}_{s}(\tau)\right)\left(\mathbf{E}_{b}\left(\tau^{\prime}\right)\right. \\
& \left.\quad+\mathbf{E}_{s}\left(\tau^{\prime}\right)\right)\left(\mathbf{E}_{b}\left(\tau^{\prime \prime}\right)+\mathbf{E}_{s}\left(\tau^{\prime \prime}\right)\right) d \tau d \tau^{\prime} d \tau^{\prime \prime}+\ldots
\end{aligned}
$$

After tedious but straightforward algebra, and neglecting terms of second order and above in the probe pulse, eq 30 can be rewritten in a series of the ground state response functions, where the probe pulse appears in the integrand only to first order (see Appendix B). This allows us to integrate over all pumpdependent variables, yielding the following formula for the dipole response

$$
\boldsymbol{\mu}(t)-\boldsymbol{\mu}_{b}(t)=\int d \tau \chi_{e f f}\left[\mathbf{E}_{b}\right](t, \tau) \mathbf{E}_{s}(\tau)
$$

As can be readily observed, this expression is equivalent to the desired eq 26, where $\boldsymbol{\mu}_{\text {ref }}=\boldsymbol{\mu}_{b}$ : the reference dipole moment that must be subtracted to eliminate all spurious signals is the dipole moment of a simulation with the pump only. This reference also been used in previous works. ${ }^{91,93}$ As can be seen in Appendix B, the pump-dependent response function $\chi_{e f f}\left[\mathbf{E}_{b}\right]$ includes its dependence to all orders on the pump pulse, keeping in mind that the linear response approximation must be valid on the probe pulse. $\chi_{\text {eff }}\left[\mathbf{E}_{b}\right]$ is equivalent to the effective response functions used in refs 91 and 94.

Fourier transforming eq 31 and making explicit the dependence on the probe time $t_{s}$

$$
\boldsymbol{\mu}\left(\omega, t_{s}\right)-\boldsymbol{\mu}_{b}\left(\omega, t_{s}\right)=\boldsymbol{\alpha}_{e f f}\left[\mathbf{E}_{b}\right]\left(\omega, t_{s}\right) \mathcal{E}_{0}^{s}
$$

where $\boldsymbol{\alpha}_{\text {eff }}=\mathcal{F}\left(\chi_{\text {eff }}\right)$ is the effective dynamic polarizability for each probe time $t_{s}$, which by assumption of the origin of the pump pulse here is equivalent to the delay time $\tau$. Finally, we arrive at an expression like eq 18 but as a function of frequency and delay time

$$
\sigma(\omega, \tau)=\frac{4 \pi \omega}{c} \operatorname{Im}\left(\alpha_{e f f}\left[\mathbf{E}_{b}\right](\omega, \tau)\right)
$$

where $\alpha_{\text {eff }}=1 / 3 \operatorname{Tr}\left[\boldsymbol{\alpha}_{\text {eff }}\right]$. After subtracting the absorption cross section of the GS, $\sigma(\omega, \tau)$ can be compared directly with experimental TA signals.

Of course, one limitation of this approach is the absence or incorrect description of electronic decay or rise lifetimes, which is one of the most interesting features that can be extracted from experimental TA signals. However, in a recent publication, we have shown for ZnTPP that important spectral features, such as the position of the ground state bleaching and excited state absorption signals, do agree with experimental data, ${ }^{21}$ which opens the door to the computational assignment of TA bands from atomistic simulations. Besides, the electronic EOM could be modified to include a damping term to account for the electronic lifetimes.

A known shortcoming in these kinds of techniques is the unphysical shift of electronic resonances in the simulated TA signals, due to the lack of memory in the adiabatic approximation for the exchange-correlation (XC) functional. ${ }^{95,96}$ The maximum frequency shift that can be observed in a particular excitation for each system depends only on the coupling of the monoelectronic transitions among the occupied molecular orbitals, which would be decoupled with a proper XC functional. This is also the reason for the wrong behavior in charge transfer processes upon resonant excitation in TDDFT. ${ }^{97}$ Nevertheless, a detailed investigation on the peak shifting has shown that its magnitude depends on the fraction of the excited population, which makes it important for small systems. ${ }^{98}$ Since TD-DFTB allows us to treat large molecules and nanostructures for these kinds of simulations, where the excited population for a reasonable laser pump pulse is not 

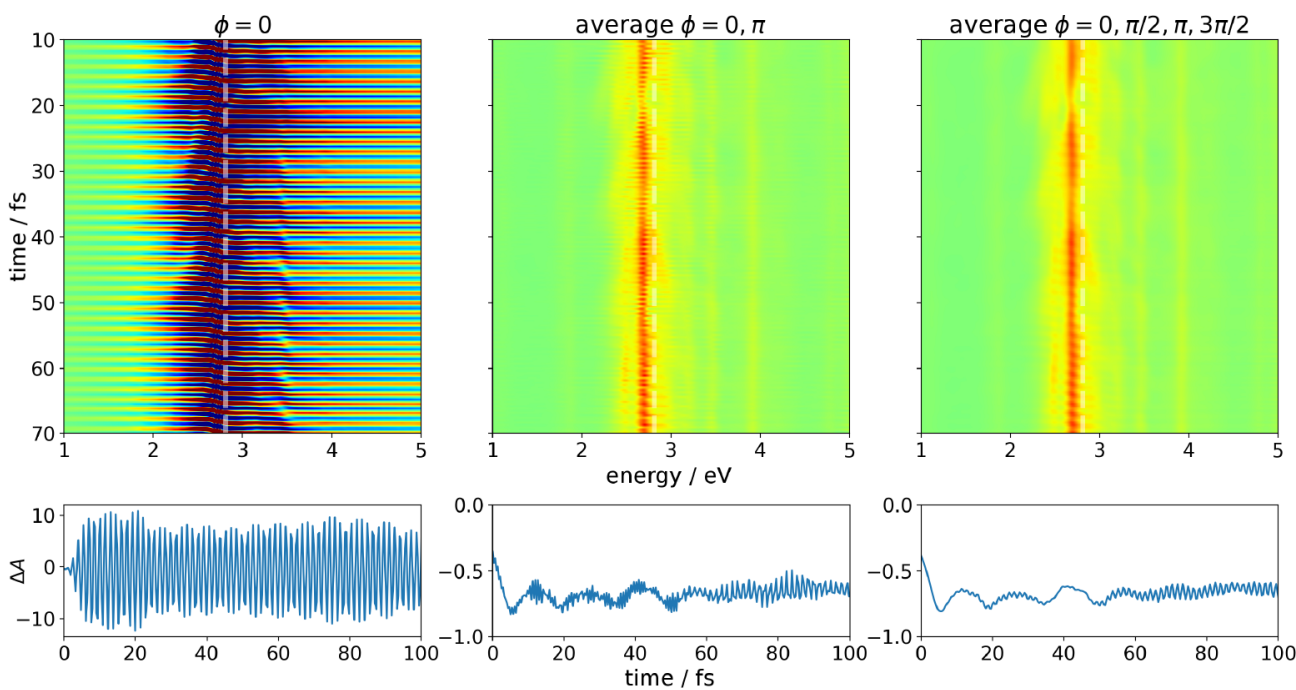

Figure 4. Transient absorption of ZnTPP (above) and time traces at $\hbar \omega=2.81 \mathrm{eV}$ using different numbers of phase differences for the average: only one phase (no average) (left), two phases (middle), and four phases (right).
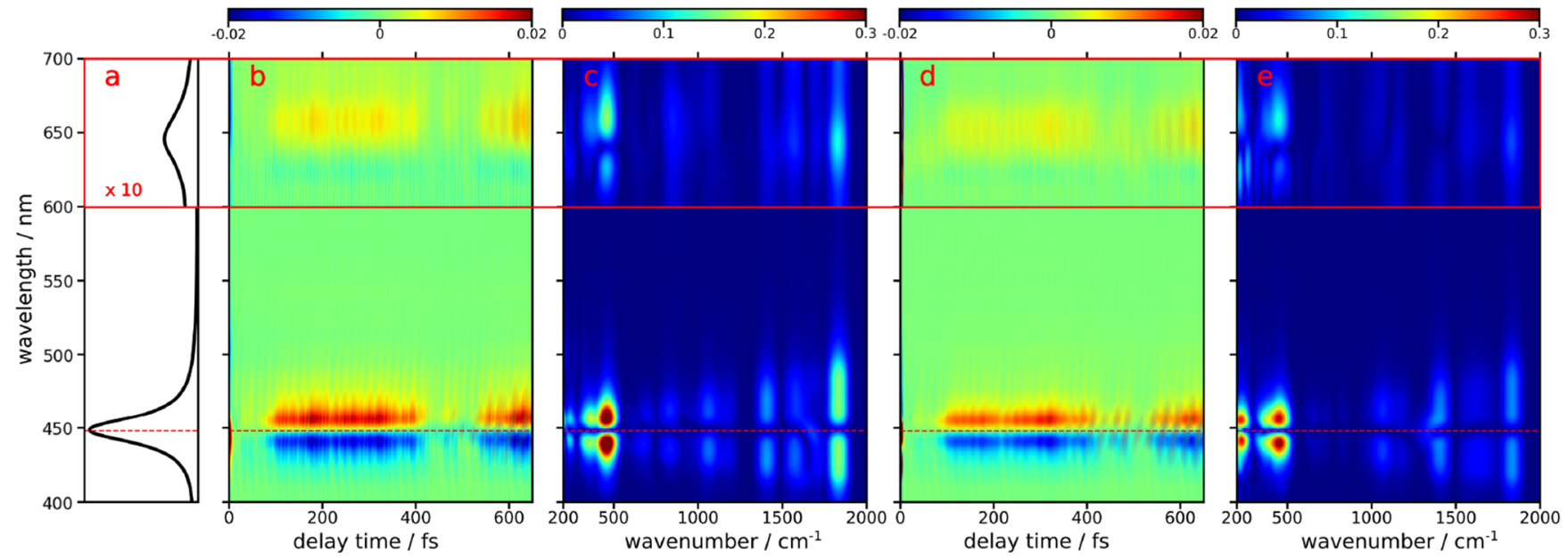

Figure 5. ZnTPP's ground state spectrum (a). Transient absorption spectra calculated by subtracting the absorption spectrum at each time with the spectrum at the time of the pulse for the pump pulse in resonance with the Soret band at $448 \mathrm{~nm}(\mathrm{~b})$ and detuned to the red at $504 \mathrm{~nm}(\mathrm{~d})$. Fourier transform of the TA spectra along the delay time axis for the TA calculated in resonance with the Soret Band (c) and detuning to the red (e). The dashed line indicates the electronic excitation (pump) wavelength.

significant with respect to the GS population, this effect is normally negligible. ${ }^{21,99}$

On the other hand, this method has the advantage of not being limited to the so-called nonoverlapping regime (when the pump and probe pulses do not overlap), but it can also be used to probe the system while the pump pulse is acting. This regime is not usually accessible by the methods reported in the literature. ${ }^{83}$ Of course, in the overlapping regime, the probe trajectory needs to include also the pump fields for the response to be consistent with the pump-only trajectory.

Equations 32 and 33 provide a recipe for the numerical simulations. Two trajectories, one with the effect of the pump only and one with the probe acting after a delay time $\tau$, must be used to compute a single TA spectrum. This procedure must be done for all desired delay times $\tau$. While the pump pulse is added to the Hamiltonian via eq 7 , the Dirac-delta probe pulse is applied to the (excited) DM analytically. ${ }^{44}$ In practice, we simulate only one pump-only trajectory and branch from it several probe trajectories, since the latter are usually shorter
(50-100 fs) than the pump trajectory (between 500 fs and 1 ps in our simulations). Therefore, the probe pulse trajectories are embarrassingly parallel. Subtracting the corresponding dipole signals, we obtain $\Delta \mu(t, \tau)$, which is damped to account for finite electronic lifetimes using an arbitrary damping parameter $\tau_{\text {damp }}$ : $\Delta \mu^{\prime}(t)=\Delta \mu(t) \exp \left(-t / \tau_{\text {damp }}\right)$.

Last but not least, classical coherent fields leave contributions in the dipole signal of eq 31 that come from the fixed relative phase between the pump and probe pulses. In experiments, these contributions are not observed since the signal is averaged incoherently over several pulses, ${ }^{83}$ unless a phase-locked experiment is done. ${ }^{100}$ By considering that the pump generates a superposition of eigenstates of the many-body Hamiltonian, Walkenhorst et al. ${ }^{94}$ showed how the initial phases of the eigenstates affect the effective response function, making the polarizability oscillate between Lorentzian and Rayleigh peaks in time, depending on the initial phases and the energy differences between the eigenstates. Thus, if this effect is not considered, the TA signal is superimposed in an oscillating signal, in which the 
frequency is that of the energy difference between the states involved in the pumped transition, as it can be seen in the Figure 4 (left) for ZnTPP after excitation of the Soret peak with a $10 \mathrm{fs}$ pump pulse. The upper panel shows the obtained TA signal, where all interesting features around the Soret energy are masked by this effect, while the lower panel shows the strong oscillatory behavior at the Soret energy.

To remove the effect of the phase difference, an average of the polarizabilities with four phase shifts between pump and probe, from 0 to $2 \pi$, must be done, as shown in ref 83 . Each phase difference, that actually is assigned to $\phi$ in eq 27 , demands a different simulation of the pump and all the corresponding probes. To see the convergence of the results when increasing the number of phases in the average, in Figure 4 (middle, right), the TA spectra of ZnTPP using two and four phases, respectively, are shown. Even when the phase average multiplies the computational cost by the number of phases, each of the sets of simulations for each phase is independent of the others and thus can be perfectly parallelized in a distributed system. Also, within TD-DFTB even for large molecular systems or small nanostructures, the computational cost is low which makes feasible such a simulation scheme.

When the phase average is not enough to get rid of all oscillatory signals, a Fourier filtering of the spectra can be done to remove all high frequencies from the signal. For example, a Gaussian filter can be applied, such that the effective polarizability is multiplied in Fourier space by a suitable Gaussian function, eliminating all the amplitudes at large frequencies, and the filtered polarizability is then reconstructed by an inverse Fourier transform.

5.2. Ultrafast Dynamics of ZnTPP On-Resonance and Off-Resonance. In a previous work, we have presented the TA spectra of zinc(II) tetraphenylporphyrin (ZnTPP) after Soretband excitation. ${ }^{21}$ In this section, we present results applying the methodology to calculate TA spectroscopy, shown in Section 5.1, for on- and off-resonance excitation of ZnTPP, and we show how it allows us to distinguish the modes that are mostly coupled to the electronic transition.

First, the GS absorption spectrum is calculated by real-time propagation after a Dirac-delta perturbation, as it is shown by eqs 17 and 18. The computed absorption spectrum is presented in Figure 5a. The two lowest-energy active bands, the Q-band at $1.93 \mathrm{eV}(642 \mathrm{~nm})$ and the Soret or B band at $2.77 \mathrm{eV}(448 \mathrm{~nm})$, are in relatively good agreement with the experimental values of 594 and $406 \mathrm{~nm}$, respectively. ${ }^{101}$

Then, ZnTPP is electronically excited by a monochromatic $\sin ^{2}$ pulse tuned to the Soret band. The pulse has a duration of $t_{p}$ $=10 \mathrm{fs}$ and peak field intensity of $0.02 \mathrm{~V}^{-1}$ (pump). The excited system evolves for $725 \mathrm{fs}$. As it is explained in Section 5.1, 1000 snapshots of the DM and geometry of the system are stored to obtain the TA spectra with a time resolution of $0.72 \mathrm{fs}$. Three trajectories after Dirac-delta kicks are run for each one of the four pump trajectories (with different phase differences) giving a total amount of 12,000 trajectories. It is worth mentioning that in this case the TA spectra are not calculated subtracting the GS spectrum, as it is usually done, since the strong bleach at the Soret band obscures the subtle changes that occur after the pump has acted. Therefore, the absorption change is calculated with respect to the spectrum at the end of the pump as shown by eq 34

$$
\Delta A(t)=A(t)-A\left(t=t_{p}\right)
$$

Figure $5 \mathrm{~b}$ and $\mathrm{d}$ show the TA spectra computed with the pump pulse tuned to the Soret band of ZnTPP at $2.77 \mathrm{eV}$ (onresonance spectra) and with the pump pulse centered at $2.46 \mathrm{eV}$, i.e., detuning the pump laser by $0.31 \mathrm{eV}$ to the red of the Soret band (off-resonance spectra), respectively. As can be observed in both figures, the signal presents oscillatory features with changes in the sidebands of the Soret band but not in the peak center, and the intensity of such oscillations is higher when the pump pulse is tuned in resonance with the Soret band. In order to get further information on these oscillatory features, the TA spectra are Fourier transformed along the delay time axis, obtaining the spectral densities shown in Figures $5 \mathrm{c}$ and e for the on-resonance and off-resonance spectra, respectively. Such spectral densities relate the absorption energy of the transient species and the energy of the different vibrational motions coupled to the ground state absorption band that is pumped. Hence, all observed peaks in the region between 200 and $2000 \mathrm{~cm}^{-1}$ in Figures $5 b$ and $d$, constitute the fingerprint of vibrational modes that are impulsively activated by the pump pulse. The spectral density is finally integrated within the pumped absorption peak region, yielding a spectrum which contains the Raman activity of the system, providing similar information to that available in the frequency domain.

This computational methodology simulates a state-of-the-art spectroscopic technique known as vibrational coherence spectroscopy (VCS), and the type of spectrum thus obtained is commonly called impulsive vibrational (IV) spectrum. ${ }^{102-108}$ VCS's main advantage over frequency domain Raman spectroscopy is that the full vibrationally coherent evolution of the system is measured. This allows us to track molecular structural dynamics accompanying ultrafast photoinduced processes in molecular systems. However, VCS has the drawback that vibrational coherences in both ground and excited states might be present, and disentangling them can be challenging.

In previous works, ${ }^{21,99}$ we have proposed interpreting the IV spectrum along with the potential energy distributed (PED) on ground state normal modes after the pulse. The PED is obtained from the pump-only trajectory, starting from the equilibrium geometry with the atoms at rest, and projecting the resulting time-resolved nuclear displacements onto normal modes coordinates as follows ${ }^{109}$

$$
Q_{i}(t)=\sum_{A} m_{A} \Delta \mathbf{r}_{A}(t) \cdot \mathbf{v}_{A i}
$$

where $\mathbf{v}_{A i}$ are the eigenvector matrix elements, $\Delta \mathbf{r}_{A}(t)$ are the nuclear displacements, $m_{A}$ is the atomic mass, and $Q_{i}$ are the coordinates in the normal mode basis set. The potential energy in the $i$ th mode can be calculated as shown in eq 36, where $c$ is the speed of light, and $\bar{\nu}_{i}$ the mode's wavenumber

$$
V_{i}(t)=\left(2 \pi c \bar{\nu}_{i} Q_{i}(t)\right)^{2}
$$

A major problem when analyzing the IV spectrum of a molecule with a large number of normal modes, such as ZnTPP (225 normal modes), is discriminating the vibrational coherences directly coupled to the electronic transition from those which are not. The solution we propose to overcome this issue is to compute two IV spectra with the exact same conditions but different pump frequency: one in resonance with the selected electronic transition and the other off-resonance (detuned to the red in this case). The differences in both IV spectra can thus be attributed to the vibrational coherences 

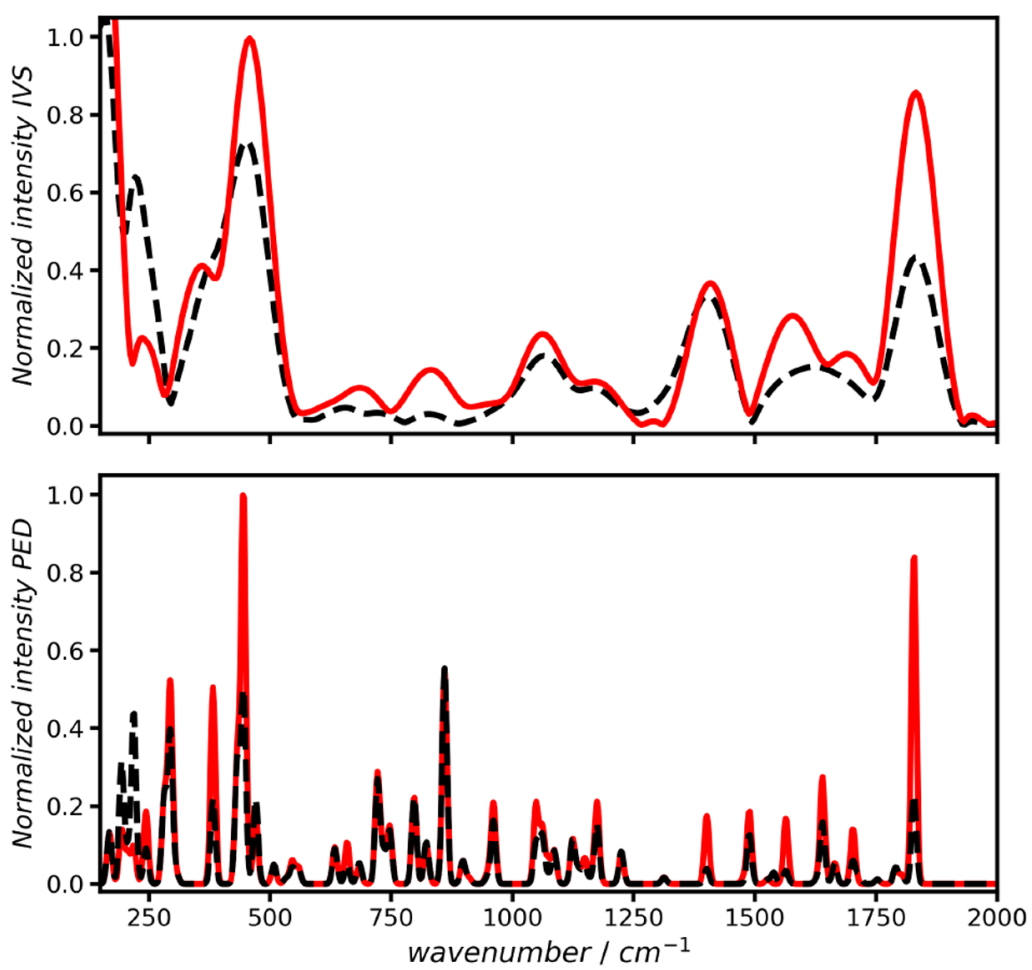

Figure 6. Impulsive vibrational spectra (top) and potential energy distributions per normal mode (bottom) of ZnTPP. The red trace is calculated with the pump laser in resonance with the Soret band, while the black trace detuning the pump laser in $0.31 \mathrm{eV}$ to the red.

directly coupled to the electron transition, and therefore, the other vibrational coherences can be filtered out.

In Figure 6 (top panel), we show the IV spectra computed onand off-resonance after the integration of the spectral densities displayed in Figures 5c and e, respectively, in comparison to the corresponding PEDs (bottom panel). We have studied the good correlation between the IV spectrum and the PED in previous works. ${ }^{21,99}$ What is different in this case, as shown in Figure 6, is the drop in intensity in both the IV spectrum and the PED for the peaks centered at 448 and $1830 \mathrm{~cm}^{-1}$. We have observed that these modes are the most coupled with the Soret transition, ${ }^{21}$ in a partial agreement with experimental evidence. ${ }^{110,111}$ These modes strongly modify the energy gap of the illuminated absorption band ${ }^{112}$ and, as we have shown, can be discriminated by direct comparison of the response at different pump energies by using the Ehrenfest TD-DFTB method. In summary, the calculation of the less computationally expensive PED does not replace the information provided by the simulated IV spectrum but rather helps to interpret, identify, and understand what normal modes are modulating the TA signal and correlate it with the symmetry of the transition density. ${ }^{21}$ The comparison of onresonance and off-resonance simulations enables us to further identify which modes are more strongly driven by the external laser.

Finally, some details about the computational resources and runtime of the calculations for this section should be discussed. Running each simulation on 4 Intel Xeon E5-2670 cores, one pump trajectory $(600,000$ steps) takes $6.5 \mathrm{~h}$, while each probe (three runs of 40,000 steps each) takes $25 \mathrm{~min}$. Since the latter are perfectly parallel, they can be run on a large number of nodes simultaneously. Using only eight machines with 16 cores each, the calculation of all 24,000 probe runs required to compute the TA spectra took 4 days in total.

\section{CONCLUSIONS}

In this work, we have explained the details of a novel implementation of electron-nuclear Ehrenfest TD-DFTB dynamics in the DFTB+ package and some important applications. We have developed a versatile, free, and opensource code to study photoinduced processes considering nuclear motion in molecular, nanoscopic, and periodic systems. The use of a semiempirical method as the base formalism increases considerably the efficiency of this implementation in comparison with ab initio methods for real-time dynamics. The ability of this tool to tackle problems previously thought to be out of the reach of atomistic quantum dynamics methods, when modest computational resources are available, has been demonstrated. First of all, by having at our disposal the underlying infrastructure developed in DFTB+ for ground state calculations, the range of possible systems that can be studied is amplified. This includes systems with correlated localized electrons through the "DFTB+U" correction and systems that are periodic in one, two, or three dimensions. Simulations of the optical properties for some of these systems have been discussed, and in some cases, they even show improvements with respect to TD-DFT. Moreover, the coupling of nuclear motion with electronic processes such as the ultrafast electron transfer in supramolecular arrangements is discussed, in this case with a structure comprising 340 atoms. A change in the charge transfer mechanism is evident by the decrease in the charge that is transferred after excitation of the acceptor. Since this is an indirect charge transfer process, the relaxation channels that the nuclear motion opens has a dramatic effect in the efficiency of the process. This can be captured at the Ehrenfest level which makes our implementation a suitable tool for future studies in the field of novel photovoltaic systems such as organic and hybrid solar cells. 
Among the most important applications of this implementation is the simulation of time-resolved and frequency-resolved excited state spectroscopies within the limitations of Ehrenfest dynamics (poorly described electronic relaxation and no electron-phonon inelastic scattering). Here, the fundamentals of transient absorption spectra simulations and their application to compute impulsive vibrational spectra are shown. While the electronic dynamics after excitation lack an accurate description of the exponential damping, the method surprisingly describes well the transient absorption signals as has been discussed in ref 21 , and the vibrational signature on the spectral signal agrees with experimental measurements. This is justified in short time scales where the electron-phonon dissipation has not developed yet, and hence, the Ehrenfest method is able to capture the electron-nuclear interactions in the quasi-adiabatic excited state regime after the pulse irradiation. This tool will most certainly be of help to the scientific community for elucidating the atomistic mechanisms in light-induced processes of relevant systems.

Regarding future developments of the tool, there are several challenges that still need to be addressed. Time-dependent perturbations along a direction in which the system is periodic would be an important feature for our tool. Higher-order corrections to the Ehrenfest methods, such as the coupled electron-ion dynamics method, can be added to improve the description of the electron-nuclear correlation, thus increasing the power of the tool to describe photochemical processes and other phenomena such as Joule heating. Moreover, the addition of open boundaries to study the dynamics of systems coupled to reservoirs will make this a new powerful tool in the field of excited state molecular electronics. In parallel to these additions, the performance of the tool will be enhanced, and the real-time dynamics of systems of more than 10,000 atoms will be within reach using distributed computing systems. With these further additions and others, we expect that this tool will open new doors for exciting discoveries in several research fields related to light-matter interactions at the molecular and nanoscopic levels.

\section{APPENDIX A: DERIVATION OF THE DENSITY MATRIX EOM}

To simplify the equations, the NAC can be thought of as an effective modification to the Hamiltonian. Let us define

$$
\tilde{H}_{\mu \nu}=H_{\mu \nu}-\mathrm{i} D_{\mu \nu}
$$

Now, to derive the EOM for the density matrix and starting from the density matrix operator $\hat{\rho}$

$$
\rho_{\nu \mu}=\sum_{i} C_{i \nu} f_{i} C_{i \mu}^{*}
$$

the derivative with respect to time leads to

$$
\begin{aligned}
& \dot{\rho}_{\mu \nu}=\sum_{i} f_{i}\left(\dot{C}_{i \nu}^{*} C_{i \mu}+C_{i \nu}^{*} \dot{C}_{i \mu}\right) \\
& \dot{\rho}_{\mu \nu}=\sum_{i} f_{i}\left\{\left(\sum_{\kappa \lambda} S_{\kappa \nu}^{-1} \tilde{H}_{\kappa \lambda}^{*} C_{i \lambda}^{*}\right) C_{i \mu}+\left(-\mathrm{i} \sum_{\eta \xi} S_{\eta \mu}^{-1} \tilde{H}_{\eta \xi} C_{i \xi}\right) C_{i \nu}^{*}\right\} \\
& \dot{\rho}_{\mu \nu}=\mathrm{i} \sum_{i} f_{i}\left\{\sum_{\kappa \lambda} S_{\kappa \nu}^{-1} \tilde{H}_{\lambda k}^{\dagger} C_{i \lambda}^{*} C_{i \mu}-\sum_{\eta \xi} S_{\eta \mu}^{-1} \tilde{H}_{\eta \xi} C_{i \xi} C_{i \nu}^{*}\right\} \\
& \dot{\rho}_{\mu \nu}=-\mathrm{i}\left\{\sum_{\eta \xi} S_{\mu \eta}^{-1} \tilde{H}_{\eta \xi} \rho_{\xi \nu}-\sum_{\kappa \lambda} \rho_{\mu \lambda} \tilde{H}_{\lambda k}^{\dagger} S_{\kappa \nu}^{-1}\right\} \\
& \dot{\rho}_{\mu \nu}=-\mathrm{i}\left(S^{-1} \tilde{H} \rho-\rho \tilde{H}^{\dagger} S^{-1}\right)_{\mu \nu}
\end{aligned}
$$

But by definition, $\tilde{H}$ is not necessarily hermitian: $\tilde{H}^{\dagger}=H+\mathrm{i} D^{\dagger}$. By substituting with this expression, we arrive at the EOM of eq 10.

\section{APPENDIX B: EFFECTIVE RESPONSE FUNCTION}

Starting from eq 30, expanding the terms between parentheses and neglecting terms of order 2 and above

$$
\begin{aligned}
\boldsymbol{\mu}(t)= & \boldsymbol{\mu}_{0}+\int d \tau \chi^{(1)}(t, \tau) \mathbf{E}_{b}(\tau)+\int d \tau \chi^{(1)}(t, \tau) \mathbf{E}_{s}(\tau) \\
+ & \frac{1}{2 !} \iint d \tau d \tau^{\prime} \chi^{(2)}\left(t, \tau, \tau^{\prime}\right) \mathbf{E}_{b}(\tau) \mathbf{E}_{b}\left(\tau^{\prime}\right) \\
+ & \frac{1}{2 !} \iint d \tau d \tau^{\prime} \chi^{(2)}\left(t, \tau, \tau^{\prime}\right) \mathbf{E}_{b}(\tau) \mathbf{E}_{s}\left(\tau^{\prime}\right) \\
& +\iint d \tau d \tau^{\prime} \chi^{(2)}\left(t, \tau, \tau^{\prime}\right) \mathbf{E}_{b}\left(\tau^{\prime}\right) \mathbf{E}_{s}(\tau) \\
& +\frac{1}{3 !} \iiint d \tau d \tau^{\prime} d \tau^{\prime \prime} \chi^{(3)}\left(t, \tau, \tau^{\prime}, \tau^{\prime \prime}\right) \mathbf{E}_{b}(\tau) \mathbf{E}_{b}\left(\tau^{\prime}\right) \mathbf{E}_{b}\left(\tau^{\prime \prime}\right) \\
+ & \frac{1}{3 !} \iiint d \tau d \tau^{\prime} d \tau^{\prime \prime} \chi^{(3)}\left(t, \tau, \tau^{\prime}, \tau^{\prime \prime}\right) \mathbf{E}_{b}(\tau) \mathbf{E}_{b}\left(\tau^{\prime}\right) \mathbf{E}_{s}\left(\tau^{\prime \prime}\right) \\
+ & \frac{1}{3 !} \iiint d \tau d \tau^{\prime} d \tau^{\prime \prime} \chi^{(3)}\left(t, \tau, \tau^{\prime}, \tau^{\prime \prime}\right) \mathbf{E}_{b}\left(\tau^{\prime}\right) \mathbf{E}_{b}\left(\tau^{\prime \prime}\right) \mathbf{E}_{s}\left(\tau^{\prime}\right) \\
+ & \frac{1}{3 !} \iiint d \tau d \tau^{\prime} d \tau^{\prime \prime} \chi^{(3)}\left(t, \tau, \tau^{\prime}, \tau^{\prime \prime}\right) \mathbf{E}_{b}\left(\tau^{\prime}\right) \mathbf{E}_{b}\left(\tau^{\prime \prime}\right) \mathbf{E}_{s}(\tau) \\
+ & \cdots
\end{aligned}
$$

By inspecting eqs 40 and 28, we see that the terms that depend only on $\mathbf{E}_{\mathrm{b}}$ are equal. Then, all these terms can be grouped in the pump-generated dipole, $\boldsymbol{\mu}_{b}(t)$, and hence, eq 40 can be rewritten as

$$
\begin{aligned}
\boldsymbol{\mu}(t) & =\boldsymbol{\mu}_{b}(t)+\int d \tau \chi^{(1)}(t, \tau) \mathbf{E}_{s}(\tau) \\
& +\frac{1}{2 !} \iint d \tau d \tau^{\prime} \chi^{(2)}\left(t, \tau, \tau^{\prime}\right) \mathbf{E}_{b}(\tau) \mathbf{E}_{s}\left(\tau^{\prime}\right) \\
& +\frac{1}{2 !} \iint d \tau d \tau^{\prime} \chi^{(2)}\left(t, \tau, \tau^{\prime}\right) \mathbf{E}_{b}\left(\tau^{\prime}\right) \mathbf{E}_{s}(\tau) \\
& +\frac{1}{3 !} \iiint d \tau d \tau^{\prime} d \tau^{\prime \prime} \chi^{(3)}\left(t, \tau, \tau^{\prime}, \tau^{\prime \prime}\right) \mathbf{E}_{b}(\tau) \mathbf{E}_{b}\left(\tau^{\prime}\right) \mathbf{E}_{s}\left(\tau^{\prime \prime}\right) \\
& +\frac{1}{3 !} \iiint d \tau d \tau^{\prime} d \tau^{\prime \prime} \chi^{(3)}\left(t, \tau, \tau^{\prime}, \tau^{\prime \prime}\right) \mathbf{E}_{b}\left(\tau^{\prime}\right) \mathbf{E}_{b}\left(\tau^{\prime \prime}\right) \mathbf{E}_{s}\left(\tau^{\prime}\right) \\
& +\frac{1}{3 !} \iiint d \tau d \tau^{\prime} d \tau^{\prime \prime} \chi^{(3)}\left(t, \tau, \tau^{\prime}, \tau^{\prime \prime}\right) \mathbf{E}_{b}\left(\tau^{\prime}\right) \mathbf{E}_{b}\left(\tau^{\prime \prime}\right) \mathbf{E}_{s}(\tau) \\
& +O\left(\left(\mathcal{E}_{0}^{s}\right)^{2}\right) \\
+ & \ldots
\end{aligned}
$$

We recognize now that all terms of order $n>1$ can be grouped as it is shown here for the second-order term 


$$
\begin{aligned}
\boldsymbol{\mu}^{(2)}(t) & =\frac{1}{2 !} \iint_{-\infty}^{\infty} d \tau d \tau^{\prime} \chi^{(2)}\left(t, \tau, \tau^{\prime}\right) \mathbf{E}_{b}(\tau) \mathbf{E}_{s}\left(\tau^{\prime}\right) \\
& +\frac{1}{2 !} \iint_{-\infty}^{\infty} d \tau d \tau^{\prime} \chi^{(2)}\left(t, \tau, \tau^{\prime}\right) \mathbf{E}_{b}\left(\tau^{\prime}\right) \mathbf{E}_{s}(\tau) \\
& \left.=\frac{1}{2 !} \int_{-\infty}^{\infty} d \tau^{\prime}\left(\int_{-\infty}^{\infty} d \tau \chi^{(2)}\left(t, \tau, \tau^{\prime}\right) \mathbf{E}_{b}(\tau)\right)\right) \mathbf{E}_{s}\left(\tau^{\prime}\right) \\
& +\frac{1}{2 !} \int_{-\infty}^{\infty} d \tau\left(d \tau^{\prime} \chi^{(2)}\left(t, \tau, \tau^{\prime}\right) \mathbf{E}_{b}\left(\tau^{\prime}\right)\right) \mathbf{E}_{s}(\tau) \\
& \left.=\frac{1}{2 !} \int_{-\infty}^{\infty} d \tau^{\prime}\left(\int_{-\infty}^{\infty} d \tau \chi^{(2)}\left(t, \tau, \tau^{\prime}\right) \mathbf{E}_{b}(\tau)\right)\right) \mathbf{E}_{s}\left(\tau^{\prime}\right) \\
& +\frac{1}{2 !} \int_{-\infty}^{\infty} d \tau\left(\int_{-\infty}^{\infty} d \tau^{\prime} \chi^{(2)}\left(t, \tau, \tau^{\prime}\right) \mathbf{E}_{b}\left(\tau^{\prime}\right)\right) \mathbf{E}_{s}(\tau)
\end{aligned}
$$

As $\chi^{(2)}\left(t, \tau, \tau^{\prime}\right)=\chi^{(2)}\left(t, \tau^{\prime}, \tau\right),{ }^{113}$ the factors between parentheses are equal (since the integrals run from $-\infty$ to $\infty$ ), which makes both integrals equal. Therefore

$$
\boldsymbol{\mu}^{(2)}(t)=2 \frac{1}{2 !} \int_{-\infty}^{\infty} d \tau \chi_{\text {eff }}^{(2)}\left[\mathbf{E}_{b}\right](t, \tau) \mathbf{E}_{s}(\tau)
$$

Similarly

$$
\boldsymbol{\mu}^{(3)}(t)=3 \frac{1}{3 !} \int_{-\infty}^{\infty} d \tau \chi_{\text {eff }}^{(3)}\left[\mathbf{E}_{b}\right](t, \tau) \mathbf{E}_{s}(\tau)
$$

and in general

$$
\boldsymbol{\mu}^{(n)}(t)=n \frac{1}{n !} \int_{-\infty}^{\infty} d \tau \chi_{\text {eff }}^{(n)}\left[\mathbf{E}_{b}\right](t, \tau) \mathbf{E}_{s}(\tau)
$$

Now, we can rewrite eq 41 as

$$
\begin{aligned}
\boldsymbol{\mu}(t) & =\boldsymbol{\mu}_{b}(t)+\int d \tau \chi^{(1)}(t, \tau) \mathbf{E}_{s}(\tau)+2 \frac{1}{2 !} \int_{-\infty}^{\infty} d \chi_{e f f}^{(2)}\left[\mathbf{E}_{b}\right](t, \tau) \mathbf{E}_{s}(\tau)+\cdots \\
\boldsymbol{\mu}(t) & =\boldsymbol{\mu}_{b}(t)+\sum_{n=1}^{\infty} \frac{1}{n !} n \int d \chi_{e f f}^{(m)}\left[\mathbf{E}_{b}\right](t, \tau) \mathbf{E}_{s}(\tau)+O\left(\left(\mathcal{E}_{0}^{s}\right)^{2}\right) \\
& =\boldsymbol{\mu}_{b}(t)+\int d \tau\left(\chi^{(1)}(t, \tau)+\sum_{n=2}^{\infty} \frac{1}{(n-1) !} \chi_{e f f}^{(m)}\left[\mathbf{E}_{b}\right](t, \tau)\right) \mathbf{E}_{s}(\tau)+O\left(\left(\mathcal{E}_{0}^{s}\right)^{2}\right) \\
& =\boldsymbol{\mu}_{b}(t)+\int d \tau \chi_{f f}\left[\mathbf{E}_{b}\right](t, \tau) \mathbf{E}_{s}(\tau)+O\left(\left(\mathcal{E}_{0}^{s}\right)^{2}\right)
\end{aligned}
$$

Rewriting this like eq 31 , one arrives at the desired expression for $\chi_{\text {eff }}$ in eq 46

$$
\begin{aligned}
\chi_{e f f}\left[\mathbf{E}_{b}\right](t, \tau) & =\chi^{(1)}(t, \tau)+\sum_{n=2}^{\infty} \frac{1}{(n-1) !} \chi_{e f f}^{(m)}\left[\mathbf{E}_{b}\right](t, \tau) \\
& =\chi^{(1)}(t, \tau)+\int d \tau^{\prime} \chi^{(2)}\left(t, \tau, \tau^{\prime}\right) \mathbf{E}_{b}\left(\tau^{\prime}\right) \\
& +\iint d \tau^{\prime} d \tau^{\prime \prime} \chi^{(3)}\left(t, \tau, \tau^{\prime}, \tau^{\prime \prime}\right) \mathbf{E}_{b}\left(\tau^{\prime}\right) \mathbf{E}_{b}\left(\tau^{\prime \prime}\right)+\ldots
\end{aligned}
$$

\section{ASSOCIATED CONTENT}

\section{(s) Supporting Information}

The Supporting Information is available free of charge at https://pubs.acs.org/doi/10.1021/acs.jctc.9b01217.

Details regarding the thermal sampling of configurations for Section 4 (PDF)

\section{AUTHOR INFORMATION}

\section{Corresponding Author}

Cristián G. Sánchez - Instituto Interdisciplinario de Ciencias Básicas, Universidad Nacional de Cuyo, CONICET, Facultad de Ciencias Exactas y Naturales, Mendoza, Argentina; ๑ orcid.org/0000-0001-7616-1802; Email: csanchez@ mendoza-conicet.gob.ar

\section{Authors}

Franco P. Bonafe - Max Planck Institute for the Structure and Dynamics of Matter, Hamburg, Germany; Universidad Nacional de Córdoba, Facultad de Ciencias Quimicas, Departamento de Quimica Teórica y Computacional, Córdoba, Argentina; Instituto de Investigaciones en Fisicoquímica de Córdoba, INFIQC (CONICET - Universidad Nacional de Córdoba), Córdoba, Argentina; ๑ orcid.org/0000-0002-2069-6776

Bálint Aradi - Bremen Center for Computational Materials Science, Universitát Bremen, Bremen, Germany

Ben Hourahine - SUPA, Department of Physics, John Anderson Building, The University of Strathclyde, Glasgow G15 6QN, United Kingdom; ๑ orcid.org/0000-0002-7667-7101

Carlos R. Medrano - Universidad Nacional de Córdoba, Facultad de Ciencias Quimicas, Departamento de Quimica Térica y Computacional, Córdoba, Argentina; Instituto de Investigaciones en Fisicoquimica de Córdoba, INFIQC (CONICET - Universidad Nacional de Córdoba), Córdoba, Argentina; ๑ orcid.org/0000-0001-7696-8366

Federico J. Hernández - Department of Physics, Universidad de Santiago de Chile, 3493 Santiago, Chile; Universidad Nacional de Córdoba, Facultad de Ciencias Quimicas, Departamento de Quimica Térica y Computacional, Córdoba, Argentina; Instituto de Investigaciones en Fisicoquimica de Córdoba, INFIQC (CONICET - Universidad Nacional de Córdoba), Córdoba, Argentina

Thomas Frauenheim - Bremen Center for Computational Materials Science, Universitát Bremen, Bremen, Germany; Computational Science Research Center (CSRC) Beijing and Computational Science and Applied Research (CSAR) Institute, Shenzhen, China

Complete contact information is available at:

https://pubs.acs.org/10.1021/acs.jctc.9b01217

\section{Notes}

The authors declare no competing financial interest.

\section{ACKNOWLEDGMENTS}

F.P.B., C.R.M., and F.J.H. thank CONICET for the doctoral and postdoctoral fellowships. The authors thank DFG-RTG2247 for the funding and the BCCMS for the computational resources provided. C.G.S. is grateful for support provided by Consejo Nacional de Investigaciones Cientificas y Técnicas (CONICET, Argentina) through Grant PIP $11220170100892 \mathrm{CO}$ and Agencia Nacional de Promoción Cientifica y Tecnológica (ANPCYT, Argentina) through Grant PICT-2017-1506. The authors thank Andrew Horsfield for the insightful discussions.

\section{REFERENCES}

(1) Curchod, B. F. E.; Rothlisberger, U.; Tavernelli, I. TrajectoryBased Nonadiabatic Dynamics with Time-Dependent Density Functional Theory. ChemPhysChem 2013, 14, 1314-1340.

(2) Crespo-Otero, R.; Barbatti, M. Recent Advances and Perspectives on Nonadiabatic Mixed Quantum-Classical Dynamics. Chem. Rev. 2018, 118, 7026-7068. 
(3) Hack, M. D.; Truhlar, D. G. Nonadiabatic Trajectories at an Exhibition. J. Phys. Chem. A 2000, 104, 7917-7926.

(4) Tully, J. C. Molecular dynamics with electronic transitions. J. Chem. Phys. 1990, 93, 1061-1071.

(5) Tapavicza, E.; Tavernelli, I.; Rothlisberger, U. Trajectory Surface Hopping within Linear Response Time-Dependent Density-Functional Theory. Phys. Rev. Lett. 2007, 98, 023001.

(6) Subotnik, J. E.; Ouyang, W.; Landry, B. R. Can we derive Tully's surface-hopping algorithm from the semiclassical quantum Liouville equation? Almost, but only with decoherence. J. Chem. Phys. 2013, 139, 214107.

(7) Kapral, R. Surface hopping from the perspective of quantumclassical Liouville dynamics. Chem. Phys. 2016, 481, 77-83.

(8) Andrade, X.; Strubbe, D.; De Giovannini, U.; Larsen, A. H.; Oliveira, M. J. T.; Alberdi-Rodriguez, J.; Varas, A.; Theophilou, I.; Helbig, N.; Verstraete, M. J.; Stella, L.; Nogueira, F.; Aspuru-Guzik, A.; Castro, A.; Marques, M. a. L.; Rubio, A. Real-space grids and the Octopus code as tools for the development of new simulation approaches for electronic systems. Phys. Chem. Chem. Phys. 2015, 17, 31371-31396.

(9) Tancogne-Dejean, N.; Oliveira, M. J. T.; Andrade, X.; Appel, H.; Borca, C. H.; Le Breton, G.; Buchholz, F.; Castro, A.; Corni, S.; Correa, A. A.; De Giovannini, U.; Delgado, A.; Eich, F. G.; Flick, J.; Gil, G.; Gomez, A.; Helbig, N.; Hübener, H.; Jestädt, R.; Jornet-Somoza, J.; Larsen, A. H.; Lebedeva, I. V.; Lüders, M.; Marques, M. A. L.; Ohlmann, S. T.; Pipolo, S.; Rampp, M.; Rozzi, C. A.; Strubbe, D. A.; Sato, S. A.; Schäfer, C.; Theophilou, I.; Welden, A.; Rubio, A. Octopus, a computational framework for exploring light-driven phenomena and quantum dynamics in extended and finite systems. J. Chem. Phys. 2020, $152,124119$.

(10) Wang, F.; Yam, C. Y.; Hu, L.; Chen, G. Time-dependent density functional theory based Ehrenfest dynamics. J. Chem. Phys. 2011, 135, 044126.

(11) Meng, S.; Kaxiras, E. Real-time, local basis-set implementation of time-dependent density functional theory for excited state dynamics simulations. J. Chem. Phys. 2008, 129, 054110.

(12) Douglas-Gallardo, O. A.; Berdakin, M.; Sánchez, C. G. Atomistic Insights into Chemical Interface Damping of Surface Plasmon Excitations in Silver Nanoclusters. J. Phys. Chem. C 2016, 120, 24389.

(13) Mansilla Wettstein, C.; Bonafé, F. P.; Oviedo, M. B.; Sánchez, C. G. Optical properties of graphene nanoflakes: Shape matters. J. Chem. Phys. 2016, 144, 224305.

(14) Bonafé, F. P.; Aradi, B.; Guan, M.; Douglas-Gallardo, O. A.; Lian, C.; Meng, S.; Frauenheim, T.; Sánchez, C. G. Plasmon-driven subpicosecond breathing of metal nanoparticles. Nanoscale 2017, 9, 12391.

(15) Oviedo, M. B.; Negre, C. F. A.; Sánchez, C. G. Dynamical simulation of the optical response of photosynthetic pigments. Phys. Chem. Chem. Phys. 2010, 12, 6706.

(16) Negre, C. F. A.; Fuertes, V. C.; Oviedo, M. B.; Oliva, F. Y.; Sánchez, C. G. Quantum Dynamics of Light-Induced Charge Injection in a Model Dye-Nanoparticle Complex. J. Phys. Chem. C 2012, 116, 14748.

(17) Medrano, C. R.; Oviedo, M. B.; Sánchez, C. G. Photoinduced Charge-Transfer Dynamics Simulations in Noncovalently Bonded Molecular Aggregates. Phys. Chem. Chem. Phys. 2016, 18, 14840.

(18) Hourahine, B.; Aradi, B.; Blum, V.; Bonafé, F.; Buccheri, A.; Camacho, C.; Cevallos, C.; Deshaye, M. Y.; Dumitrică, T.; Dominguez, A.; Ehlert, S.; Elstner, M.; van der Heide, T.; Hermann, J.; Irle, S.; Kranz, J. J.; Köhler, C.; Kowalczyk, T.; Kubař, T.; Lee, I. S.; Lutsker, V.; Maurer, R. J.; Min, S. K.; Mitchell, I.; Negre, C.; Niehaus, T. A.; Niklasson, A. M. N.; Page, A. J.; Pecchia, A.; Penazzi, G.; Persson, M. P.; Řezáč, J.; Sánchez, C. G.; Sternberg, M.; Stöhr, M.; Stuckenberg, F.; Tkatchenko, A.; Yu, V. W.; Frauenheim, T. DFTB+, a software package for efficient approximate density functional theory based atomistic simulations. J. Chem. Phys. 2020, 152, 124101.

(19) Hourahine, B.; Sanna, S.; Aradi, B.; Köhler, C.; Niehaus, T.; Frauenheim, T. Self-interaction and strong correlation in DFTB. J. Phys. Chem. A 2007, 111, 5671-5677.
(20) Gaus, M.; Cui, Q.; Elstner, M. DFTB3: extension of the selfconsistent-charge density-functional tight-binding method (SCCDFTB). J. Chem. Theory Comput. 2011, 7, 931-948.

(21) Bonafé, F. P.; Hernández, F. J.; Aradi, B.; Frauenheim, T.; Sánchez, C. G. Fully Atomistic Real-Time Simulations of Transient Absorption Spectroscopy. J. Phys. Chem. Lett. 2018, 9, 4355.

(22) Elstner, M.; Porezag, D.; Jungnickel, G.; Elsner, J.; Haugk, M.; Frauenheim, T.; Suhai, S.; Seifert, G. Self-consistent-charge densityfunctional tight-binding method for simulations of complex materials properties. Phys. Rev. B: Condens. Matter Mater. Phys. 1998, 58, 7260.

(23) Frauenheim, T.; Seifert, G.; Elstner, M.; Niehaus, T.; Köhler, C.; Amkreutz, M.; Sternberg, M.; Hajnal, Z.; Carlo, A.; Suhai, S. Atomistic simulations of complex materials: ground-state and excited-state properties. J. Phys.: Condens. Matter 2002, 14, 3015.

(24) Koskinen, P.; Mäkinen, V. Density-functional tight-binding for beginners. Comput. Mater. Sci. 2009, 47, 237.

(25) Bodrog, Z.; Aradi, B. Possible improvements to the selfconsistent-charges density-functional tight-binding method within the second order. Phys. Status Solidi B 2012, 249, 259-269.

(26) Domínguez, A.; Aradi, B.; Frauenheim, T.; Lutsker, V.; Niehaus, T. A. Extensions of the Time-Dependent Density Functional Based Tight-Binding Approach. J. Chem. Theory Comput. 2013, 9, 49014914.

(27) Domínguez, A.; Niehaus, T. A.; Frauenheim, T. Accurate hydrogen bond energies within the density functional tight binding method. J. Phys. Chem. A 2015, 119, 3535-3544.

(28) Boleininger, M.; Guilbert, A. A.; Horsfield, A. P. Gaussian polarizable-ion tight binding. J. Chem. Phys. 2016, 145, 144103.

(29) Boleininger, M.; Horsfield, A. P. Efficient local-orbitals based method for ultrafast dynamics. J. Chem. Phys. 2017, 147, 044111.

(30) Niehaus, T. Approximate time-dependent density functional theory. J. Mol. Struct.: THEOCHEM 2009, 914, 38.

(31) Oviedo, M. B.; Sánchez, C. G. Transition Dipole Moments of the Qy Band in Photosynthetic Pigments. J. Phys. Chem. A 2011, 115, 12280

(32) Todorov, T. N. Time-dependent tight binding. J. Phys.: Condens. Matter 2001, 13, 10125.

(33) Niehaus, T. A.; Heringer, D.; Torralva, B.; Frauenheim, T. Importance of electronic self-consistency in the TDDFT based treatment of nonadiabatic molecular dynamics. Eur. Phys. J. D 2005, 35, 467.

(34) Runge, E.; Gross, E. K. Density-functional theory for timedependent systems. Phys. Rev. Lett. 1984, 52, 997.

(35) Breuer, H.-P.; Petruccione, F. The Theory of Open Quantum Systems; Oxford University Press: Oxford, 2010.

(36) Oviedo, M. B.; Zarate, X.; Negre, C. F. a.; Schott, E.; ArratiaPérez, R.; Sánchez, C. G. Quantum dynamical simulations as a tool for predicting photoinjection mechanisms in dye-sensitized $\mathrm{TiO} 2$ solar cells. J. Phys. Chem. Lett. 2012, 3, 2548.

(37) Kürpick, P.; Sepp, W.-D.; Fricke, B. Relativistic ab initio description of the $\mathrm{K}$-vacancy production in heavy-ion-atom collision systems with solid targets. Phys. Rev. A: At., Mol., Opt. Phys. 1995, 51, 3693-3697.

(38) Horsfield, A. P.; Bowler, D. R.; Fisher, A. J.; Todorov, T. N.; Montgomery, M. J. Power dissipation in nanoscale conductors: classical, semi-classical and quantum dynamics. J. Phys.: Condens. Matter 2004, 16, 3609.

(39) Montgomery, M. J.; Todorov, T. N.; Sutton, a. P. Power dissipation in nanoscale conductors. J. Phys.: Condens. Matter 2002, 14, 5377.

(40) Haug, H.; Jauho, A.-P. Quantum Kinetics in Transport and Optics of Semiconductors; Solid-State Sciences; Springer: Berlin, Heidelberg, 2008; Vol. 123.

(41) McEniry, E. J.; Frederiksen, T.; Todorov, T. N.; Dundas, D.; Horsfield, A. P. Inelastic quantum transport in nanostructures: The selfconsistent Born approximation and correlated electron-ion dynamics. Phys. Rev. B: Condens. Matter Mater. Phys. 2008, 78, 035446.

(42) Lin, Z.; Allen, R. E. Ultrafast equilibration of excited electrons in dynamical simulations. J. Phys.: Condens. Matter 2009, 21, 485503. 
(43) Yabana, K.; Bertsch, G. Time-dependent local-density approximation in real time. Phys. Rev. B: Condens. Matter Mater. Phys. 1996, 54, 4484-4487.

(44) Negre, C. F. A. Dinámica Cuántica de Nanosistemas Fuera del Equilibrio. Ph.D. thesis, Universidad Nacional De Córdoba, Argentina, 2010.

(45) Castro, A.; Appel, H.; Oliveira, M.; Rozzi, C. A.; Andrade, X.; Lorenzen, F.; Marques, M. A. L.; Gross, E. K. U.; Rubio, A. Octopus: a Tool for the Application of Time-Dependent Density Functional Theory. Phys. Status Solidi B 2006, 243, 2465-2488.

(46) Onida, G.; Reining, L.; Rubio, A. Electronic excitations: densityfunctional versus many-body Green's-function approaches. Rev. Mod. Phys. 2002, 74, 601-659.

(47) Fuertes, V. C.; Negre, C. F. a.; Oviedo, M. B.; Bonafé, F. P.; Oliva, F. Y.; Sánchez, C. G. A theoretical study of the optical properties of nanostructured TiO 2. J. Phys.: Condens. Matter 2013, 25, 115304.

(48) Mansilla Wettstein, C.; Sánchez, C. G. Characterization of $\mathrm{ZnO}$ as substrate for DSSC. Phys. Chem. Chem. Phys. 2018, 20, 21910.

(49) Negre, C. F. a.; Perassi, E. M.; Coronado, E. a.; Sánchez, C. G. Quantum dynamical simulations of local field enhancement in metal nanoparticles. J. Phys.: Condens. Matter 2013, 25, 125304.

(50) Aradi, B.; Hourahine, B.; Frauenheim, T. DFTB+, a Sparse Matrix-Based Implementation of the DFTB Method. J. Phys. Chem. A 2007, 111, 5678.

(51) Swope, W. C.; Andersen, H. C.; Berens, P. H.; Wilson, K. R. A computer simulation method for the calculation of equilibrium constants for the formation of physical clusters of molecules: Application to small water clusters. J. Chem. Phys. 1982, 76, 637-649.

(52) Novoselov, K. S. Electric Field Effect in Atomically Thin Carbon Films. Science 2004, 306, 666-669.

(53) Chen, J. H.; Jang, C.; Xiao, S.; Ishigami, M.; Fuhrer, M. S. Intrinsic and extrinsic performance limits of graphene devices on $\mathrm{SiO} 2$. Nat. Nanotechnol. 2008, 3, 206-209.

(54) Schwierz, F. Graphene transistors. Nat. Nanotechnol. 2010, 5, 487-496.

(55) Son, Y.-W.; Cohen, M. L.; Louie, S. G. Energy Gaps in Graphene Nanoribbons. Phys. Rev. Lett. 2006, 97, 216803.

(56) Son, Y.-W.; Cohen, M. L.; Louie, S. G. Half-metallic graphene nanoribbons. Nature 2006, 444, 347-349.

(57) Wang, W.-X.; Zhou, M.; Li, X.; Li, S.-Y.; Wu, X.; Duan, W.; He, L. Energy gaps of atomically precise armchair graphene sidewall nanoribbons. Phys. Rev. B: Condens. Matter Mater. Phys. 2016, 93, 241403.

(58) Elstner, M.; Porezag, D.; Jungnickel, G.; Elsner, J.; Haugk, M.; Frauenheim, T.; Suhai, S.; Seifert, G. Self-consistent-charge densityfunctional tight-binding method for simulations of complex materials properties. Phys. Rev. B: Condens. Matter Mater. Phys. 1998, 58, 72607268.

(59) Son, Y.-W.; Cohen, M. L.; Louie, S. G. Energy Gaps in Graphene Nanoribbons. Phys. Rev. Lett. 2006, 97, 216803-4.

(60) Lian, C.; Guan, M.; Hu, S.; Zhang, J.; Meng, S. Photoexcitation in Solids: First-Principles Quantum Simulations by Real-Time TDDFT. Adv. Theory Simul. 2018, 1, 1800055-10.

(61) Yang, L.; Deslippe, J.; Park, C.-H.; Cohen, M. L.; Louie, S. G. Excitonic Effects on the Optical Response of Graphene and Bilayer Graphene. Phys. Rev. Lett. 2009, 103, 186802-4.

(62) Orhan, O. K.; O’Regan, D. D. TDDFT + U: A critical assessment of the Hubbard $U$ correction to exchange-correlation kernels and potentials. Phys. Rev. B: Condens. Matter Mater. Phys. 2019, 99, 165120.

(63) Pierloot, K.; Tsokos, E.; Vanquickenborne, L. Optical spectra of $\mathrm{Ni}(\mathrm{CO})_{4}$ and $\mathrm{Cr}(\mathrm{CO})_{6}$ revisited. J. Phys. Chem. 1996, 100, 1654516550.

(64) Anisimov, V. I.; Aryasetiawan, F.; Lichtenstein, A. I. Firstprinciples calculations of the electronic structure and spectra of strongly correlated systems: the LDA $+U$ method. J. Phys.: Condens. Matter 1997, 9, 767-808.

(65) Dudarev, S. L.; Botton, G. A.; Savrasov, S. Y.; Humphreys, C. J.; Sutton, A. P. Electron-energy-loss spectra and the structural stability of nickel oxide: An LSDA+U study. Phys. Rev. B: Condens. Matter Mater. Phys. 1998, 57, 1505-1509.

(66) Han, M. J.; Ozaki, T.; Yu, J. O(N) LDA + U electronic structure calculation method based on the nonorthogonal pseudoatomic orbital basis. Phys. Rev. B: Condens. Matter Mater. Phys. 2006, 73, 045110.

(67) Petukhov, A. G.; Mazin, I. I.; Chioncel, L.; Lichtenstein, A. I. Correlated metals and the LDA $+U$ method. Phys. Rev. B: Condens. Matter Mater. Phys. 2003, 67, 153106.

(68) Hourahine, B.; Sanna, S.; Aradi, B.; Köhler, C.; Niehaus, T.; Frauenheim, T. Self-Interaction and Strong Correlation in DFTB. J. Phys. Chem. A 2007, 111, 5671-5677.

(69) Zheng, G.; Witek, H. A.; Bobadova-Parvanova, P.; Irle, S.; Musaev, D. G.; Prabhakar, R.; Morokuma, K.; Lundberg, M.; Elstner, M.; Köhler, C.; Frauenheim, T. Parameter Calibration of TransitionMetal Elements for the Spin-Polarized Self-Consistent-Charge DensityFunctional Tight-Binding (DFTB) Method: Sc, Ti, Fe, Co, and Ni. J. Chem. Theory Comput. 2007, 3, 1349-1367.

(70) Jacob, C. R.; Reiher, M. Spin in density-functional theory. Int. J. Quantum Chem. 2012, 112, 3661-3684.

(71) Oppenheim, J. J.; McNicholas, B. J.; Miller, J.; Gray, H. B. Electronic Structure of Tetracyanonickelate(II). Inorg. Chem. 2019, 58, 15202-15206.

(72) Filippetti, A.; Spaldin, N. A. Self-interaction-corrected pseudopotential scheme for magnetic and strongly-correlated systems. Phys. Rev. B: Condens. Matter Mater. Phys. 2003, 67, 125109.

(73) Pemmaraju, C. D.; Archer, T.; Sánchez-Portal, D.; Sanvito, S. Atomic-orbital-based approximate self-interaction correction scheme for molecules and solids. Phys. Rev. B: Condens. Matter Mater. Phys. 2007, 75, 045101.

(74) Falke, S. M.; Rozzi, C. A.; Brida, D.; Maiuri, M.; Amato, M.; Sommer, E.; De Sio, A.; Rubio, A.; Cerullo, G.; Molinari, E.; Lienau, C. Coherent ultrafast charge transfer in an organic photovoltaic blend. Science 2014, 344, 1001.

(75) Rozzi, C. A.; Falke, S. M.; Spallanzani, N.; Rubio, A.; Molinari, E.; Brida, D.; Maiuri, M.; Cerullo, G.; Schramm, H.; Christoffers, J.; Lienau, C. Quantum coherence controls the charge separation in a prototypical artificial light-harvesting system. Nat. Commun. 2013, 4, 1602.

(76) Long, R.; Prezhdo, O. V. Quantum Coherence Facilitates Efficient Charge Separation at a $\mathrm{MoS}_{2} / \mathrm{MoSe}_{2}$ van der Waals Junction. Nano Lett. 2016, 16, 1996-2003.

(77) Zheng, Q.; Saidi, W. A.; Xie, Y.; Lan, Z.; Prezhdo, O. V.; Petek, H.; Zhao, J. Phonon-Assisted Ultrafast Charge Transfer at van der Waals Heterostructure Interface. Nano Lett. 2017, 17, 6435-6442.

(78) Medrano, C. R.; Sánchez, C. G. Trap-Door-Like Irreversible Photoinduced Charge Transfer in a Donor-Acceptor Complex. J. Phys. Chem. Lett. 2018, 9, 3517-3524.

(79) Pollard, W. T.; Mathies, R. A. Analysis of femtosecond dynamic absorption spectra of nonstationary states. Annu. Rev. Phys. Chem. 1992, 43, 497.

(80) Mukamel, S. Principles of Nonlinear Optical Spectroscopy; Oxford University Press, 1999; p 576.

(81) Finkelstein, G.; Bar-Ad, S.; Carmel, O.; Bar-Joseph, I.; Levinson, $Y$. Biexcitonic effects in transient nonlinear optical experiments in quantum wells. Phys. Rev. B: Condens. Matter Mater. Phys. 1993, 47, 12964.

(82) Tanimura, Y.; Mukamel, S. Femtosecond pump-probe spectroscopy of intermolecular vibrations in molecular dimers. J. Chem. Phys. 1995, 103, 1981.

(83) Seidner, L.; Stock, G.; Domcke, W. Nonperturbative approach to femtosecond spectroscopy: General theory and application to multidimensional nonadiabatic photoisomerization processes. J. Chem. Phys. 1995, 103, 3998.

(84) Domcke, W.; Stock, G. Theory of Ultrafast Nonadiabatic Excited-State Processes and their Spectroscopic Detection in Real Time. In Advances in Chemical Physics; 1997; Vol. 100, Chapter 1. DOI: 10.1002/9780470141595.ch1.

(85) Bratos, S.; Leicknam, J.-C.; Borgis, D.; Staib, A. Subpicosecond pump-probe absorption of the hydrated electron: Nonlinear response 
theory and computer simulation. Phys. Rev. E: Stat. Phys., Plasmas, Fluids, Relat. Interdiscip. Top. 1997, 55, 7217.

(86) Kumar, A. T.; Rosca, F.; Widom, A.; Champion, P. M. Investigations of amplitude and phase excitation profiles in femtosecond coherence spectroscopy. J. Chem. Phys. 2001, 114, 701.

(87) Tortschanoff, A.; Mukamel, S. Pump-probe simulation study of the two-exciton manifold of dendrimers. J. Phys. Chem. A 2002, 106, 7521.

(88) Gelin, M. F.; Pisliakov, A. V.; Egorova, D.; Domcke, W. A simple model for the calculation of nonlinear optical response functions and femtosecond time-resolved spectra. J. Chem. Phys. 2003, 118, 5287.

(89) Sugisaki, M.; Yanagi, K.; Cogdell, R. J.; Hashimoto, H. Unified explanation for linear and nonlinear optical responses in $\beta$-carotene: $\mathrm{A}$ sub-20-fs degenerate four-wave mixing spectroscopic study. Phys. Rev. B: Condens. Matter Mater. Phys. 2007, 75, 155110.

(90) Polli, D.; Brida, D.; Mukamel, S.; Lanzani, G.; Cerullo, G. Effective temporal resolution in pump-probe spectroscopy with strongly chirped pulses. Phys. Rev. A: At., Mol., Opt. Phys. 2010, 82, 053809.

(91) De Giovannini, U.; Brunetto, G.; Castro, A.; Walkenhorst, J.; Rubio, A. Simulating pump-probe photoelectron and absorption spectroscopy on the attosecond timescale with time-dependent density functional theory. ChemPhysChem 2013, 14, 1363.

(92) Boleininger, M. Gaussian Tight Binding Study of Ultrafast Electron Dynamics. Ph.D. thesis, Imperial College, London, 2017.

(93) Fischer, S. A.; Cramer, C. J.; Govind, N. Excited State Absorption from Real-Time Time-Dependent Density Functional Theory. J. Chem. Theory Comput. 2015, 11, 4294.

(94) Walkenhorst, J.; De Giovannini, U.; Castro, A.; Rubio, A. Tailored pump-probe transient spectroscopy with time-dependent density-functional theory: controlling absorption spectra. Eur. Phys. J. B 2016, 89, na DOI: 10.1140/epjb/e2016-70064-0.

(95) Elliott, P.; Maitra, N. T. Propagation of initially excited states in time-dependent density-functional theory. Phys. Rev. A: At., Mol., Opt. Phys. 2012, 85, 052510.

(96) Fuks, J. I.; Luo, K.; Sandoval, E. D.; Maitra, N. T. Time-Resolved Spectroscopy in Time-Dependent Density Functional Theory: An Exact Condition. Phys. Rev. Lett. 2015, 114, 183002.

(97) Fuks, J. I. Time-dependent density functional theory for chargetransfer dynamics: review of the causes of failure and success*. Eur. Phys. J. B 2016, 89, 236.

(98) Provorse, M. R.; Habenicht, B. F.; Isborn, C. M. Peak-Shifting in Real-Time Time-Dependent Density Functional Theory. J. Chem. Theory Comput. 2015, 11, 4791.

(99) Hernández, F. J.; Bonafé, F. P.; Aradi, B.; Frauenheim, T.; Sánchez, C. G. Simulation of Impulsive Vibrational Spectroscopy. J. Phys. Chem. A 2019, 123, 2065-2072.

(100) Cho, M.; Scherer, N. F.; Fleming, G. R.; Mukamel, S. Photon echoes and related four-wave-mixing spectroscopies using phase-locked pulses. J. Chem. Phys. 1992, 96, 5618.

(101) Edwards, L.; Dolphin, D.; Gouterman, M.; Adler, A. Porphyrins XVII. Vapor Absorption Spectra and Redox Reactions: Tetraphenylporphins and Porphin. J. Mol. Spectrosc. 1971, 38, 16.

(102) Collini, E.; Wong, C. Y.; Wilk, K. E.; Curmi, P. M. G.; Brumer, P.; Scholes, G. D. Coherently wired light-harvesting in photosynthetic marine algae at ambient temperature. Nature 2010, 463, 644-647.

(103) Scholes, G. D.; Fleming, G. R.; Olaya-Castro, A.; van Grondelle, R. Lessons from nature about solar light harvesting. Nat. Chem. 2011, 3, 763-774.

(104) Huelga, S.; Plenio, M. Vibrations, quanta and biology. Contemp. Phys. 2013, 54, 181-207.

(105) Fuller, F. D.; Pan, J.; Gelzinis, A.; Butkus, V.; Senlik, S. S.; Wilcox, D. E.; Yocum, C. F.; Valkunas, L.; Abramavicius, D.; Ogilvie, J. P. Vibronic coherence in oxygenic photosynthesis. Nat. Chem. 2014, 6, 706-711.

(106) Romero, E.; Augulis, R.; Novoderezhkin, V. I.; Ferretti, M.; Thieme, J.; Zigmantas, D.; van Grondelle, R. Quantum coherence in photosynthesis for efficient solar-energy conversion. Nat. Phys. 2014, $10,676-682$.
(107) Scholes, G. D.; Fleming, G. R.; Chen, L. X.; Aspuru-Guzik, A.; Buchleitner, A.; Coker, D. F.; Engel, G. S.; van Grondelle, R.; Ishizaki, A.; Jonas, D. M.; Lundeen, J. S.; McCusker, J. K.; Mukamel, S.; Ogilvie, J. P.; Olaya-Castro, A.; Ratner, M. A.; Spano, F. C.; Whaley, K. B.; Zhu, $\mathrm{X}$. Using coherence to enhance function in chemical and biophysical systems. Nature 2017, 543, 647-656.

(108) Gueye, M.; Manathunga, M.; Agathangelou, D.; Orozco, Y.; Paolino, M.; Fusi, S.; Haacke, S.; Olivucci, M.; Léonard, J. Engineering the vibrational coherence of vision into a synthetic molecular device. Nat. Commun. 2018, 9, 313.

(109) Horiuchi, T.; Go, N. Projection of Monte Carlo and Molecular Dynamics Trajectories onto the Normal Modes Axes. Proteins: Struct., Funct., Genet. 1991, 10, 106.

(110) Abraham, B.; Nieto-Pescador, J.; Gundlach, L. Ultrafast Relaxation Dynamics of Photoexcited Zinc-Porphyrin: ElectronicVibrational Coupling. J. Phys. Chem. Lett. 2016, 7, 3151.

(111) Yoon, M. C.; Jeong, D. H.; Cho, S.; Kim, D.; Rhee, H.; Joo, T. Ultrafast transient dynamics of $\mathrm{Zn}(\mathrm{II})$ porphyrins: Observation of vibrational coherence by controlling chirp of femtosecond pulses. J. Chem. Phys. 2003, 118, 164.

(112) Liebel, M.; Schnedermann, C.; Bassolino, G.; Taylor, G.; Watts, A.; Kukura, P. Direct Observation of the Coherent Nuclear Response after the Absorption of a Photon. Phys. Rev. Lett. 2014, 112, 238301.

(113) Parr, R. G.; Yang, W. Density-Functional Theory of Atoms and Molecules; Oxford University Press, 1989; p 333. 